VarSITI - Variability of the Sun and Its Terrestrial Impacts

\title{
VarSITI Completion General Symposium
}
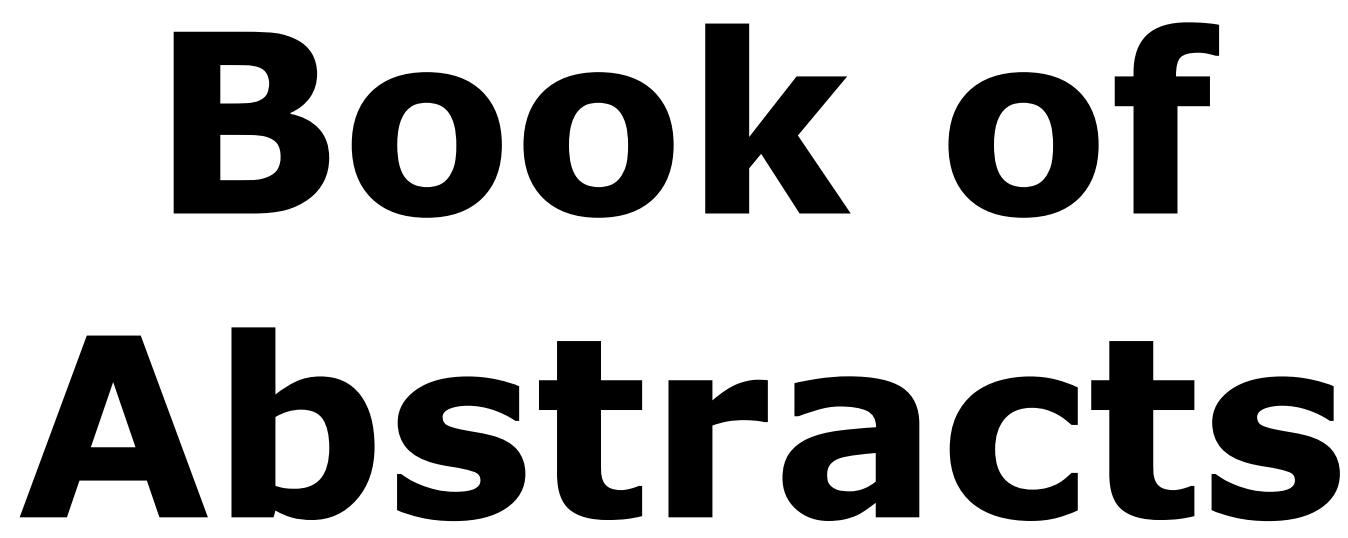

\section{0-14 June, 2019, Sofia, Bulgaria}




\title{
Scientific Organizing Committee
}

Jacob Bortnik, Dept. of Atmospheric and Oceanic Sciences, UCLA, USA Aude Chambodut, Institut de Physique du Globe, Université de Strasbourg, France

Ioannis A. Daglis, University of Athens and National Observatory of Athens, Greece

Katya Georgieva, Space Research and Technologies Institute (SRTI), Bulgaria (co-chair)

Nat Gopalswamy, Heliophysics Division, NASA/GSFC, USA

Shri Kanekal, NASA/GSFC, Greenbelt, USA

Emilia Kilpua, Department of Physics, University of Helsinki, Finland Jan Lastovicka, Institute of Atmospheric Physics, Prague, Czech Republic Franz-Josef Lübken, Leibniz-Institute of Atmospheric Physics, Kühlungsborn, Germany Dan Marsh, NCAR, Boulder, USA

Petrus Martens, Georgia State University, USA

Yoshi Miyoshi, ISEE, Nagoya University, Japan

Kalevi Mursula, University of Oulu, Finland

Dibyendu Nandi, Indian Institute of Science Education and Research (IISER), Kolkata, India

Vladimir Obridko, IZMIRAN, Russia

Craig Rodger, University of Otago, New Zealand

Annika Seppälä, Finnish Meteorological Institute, Finland

Kazuo Shiokawa, Solar-Terrestrial Environment Laboratory (STEL), Nagoya U, Japan (co-chair)

Manuela Temmer, University of Graz, Austria

Ilya Usoskin, University of Oulu, Finland

William Ward, University of New Brunswick, Canada

David Webb, Boston College, USA

Jie Zhang, George Mason University, USA

\section{Local Organizing Committee}

from Space Research and Technology Institute, Bulgarian Academy of Science

\author{
Boian Kirov (chair) \\ Dimitar Danov \\ Rositsa Miteva \\ Simeon Asenovski \\ Peter Tonev
}

The present workshop is organized with the support of the National Science Fund of Bulgaria, grant No. КП-06-МНФ/8. 


\section{CONTENTS}

Adebesin B.O., Adeniyi J.O., Adebiyi S.J., Ikubanni S.O., Dependence of equatorial ionization gradient with Sq current and F2-layer bottomside thickness parameter in the African region

Adebiyi S.J., Adeniyi J.O., Adebesin B.O., Ikubanni S.O., Pattern of digisonde measured-scale height derived at an African equatorial station

Alexakis P., Mavromichalaki H., Geoeffectiveness of the interplanetary coronal mass ejections over the years 1996-2017

Belakhovsky V.B., Shiokawa K., Matsuoka A., Kasahara Y., Shinohara I., Miyoshi Y., The long-lasting QP emissions observed on ERG satellite and Lovozero station

Belakhovsky V.B., Shiokawa K., Matsuoka A., Wang S.-Y., Kazama Y., Tam S., Kasahara S., Yokota S., Keika K., Hori T., Shinohara I., Miyoshi Y., The generation of monochromatic Pc4 pulsations by the electron cloud

Belehaki A., and TechTIDE EC consortium, Real-time identification of travelling ionospheric disturbances and validation methodologies

Borisenko A.V., Podgorny I.M., Podgorny A.I., Using CUDA supercomputing for acceleration of calculations in study of solar flare mechanism for coronal MHD simulation

Brun A.S., Strugarek A., The evolving solar-stellar dynamo

04

Clette $F_{.}$, Lefèvre $L_{.}$, Reconciling the sunspot and group number series

Dasso S., State of the art and gaps on the role of interplanetary structures on Sun-Earth coupling

Dasso S., Gulisano A.M., Areso O., Pereira M., et al., for the LAGO collaboration, First observations from the space weather antarctic node of the Latin American Giant Observatory (LAGO)

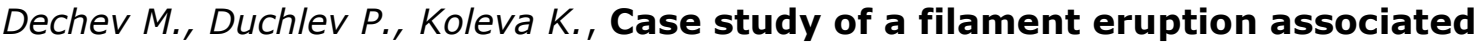
with a C8.4 flare

Demetrescu Cr., Dobrica V., Georgieva K., Kirov B., External drivers of decadal variations in the geomagnetic field and Earth's rotation

Desai M.I., Dayeh M.A., Ebert R.W., Energetic particles in the heliosphere and their impacts on geospace

Despirak I.V., Kleimenova N.G., Gromova L.I., Gromov S.V., Malysheva L.M.,

Space weather event on 7-8 September 2017: Magnetic supersubstorms and dayside polar substorm

Despirak I.V., Kozelova T.V., Kozelov B.V., Lubchich A.A., THEMIS and groundbased observations during event on 24 December 2014

Dikpati M., Advances and limitations in physics-based predictions of shortterm and decadal solar activity

Dobrica V., Demetrescu Cr., Greculeasa R., Stefan Cr., Surface electric field over Europe as hazardous response to the intense/severe geomagnetic storms of the solar cycle 24

Duchlev P., Dechev M., Koleva K., Eruptions of two coupled flux ropes of a filament and associated ribbon flare and CME 
Duggirala P., Singh R.P., Mandal S., Keran D.K., Laskar F.I., Investigation on upper atmospheric variability on a global scale due to influence of solar forcing from above and vertical coupling from below

Dumbovic M., Utilizing galactic cosmic rays to understand the Sun-to-Earth evolution of CMEs

Figueiredo C.A.O.B., Takahashi H., Wrasse C.M., Otsuka Y., Shiokawa K., Buriti R.A., Paulino $I .$, Barros D., Tilted equatorial plasma bubble and plasma blobs after interaction with medium-scale traveling ionospheric disturbances and midnight brightness wave

Funke $B$., Towards a better understanding of EPP effects on the middle atmosphere and climate

Gavrilov N.M., Kshevetskii S.P., Dynamical and thermal effects of nonlinear acoustic-gravity waves in the upper atmosphere at high and low solar activity

Génot $V_{\text {., }}$ and the CDPP team, Data management, analysis tools and science applications by the Plasma Physics Data Center (CDPP)

Gopalswamy N., Akiyama S., Mäkelä P., Yashiro S., Xie H., Solar energetic particle event associated with the 2017 September 6 coronal mass ejection

Gopalswamy N., Mäkelä P., Yashiro S., Akiyama S., A catalog of interplanetary type II radio bursts observed by the Wind/WAVES instrument

Gopalswamy N., Yashiro S., Mäkelä P., Akiyama S., Predicting the solar-cycle strength from the polar brightness: Contribution from the Nobeyama Radioheliograph

Guineva V., Despirak I.V., Kleimenova N., Werner R., Substorm activity at high and middle latitudes during large geomagnetic storms

Hazra S., Brun A.S., Buchlin E., Reville V., Strugarek A., Perri B., Effect of solar variability on the solar wind during solar cycle 23 and 24

Horne R.B., The predictability of the Earth's radiation belts

Ikubanni S.O., Adebiyi S.J., Adebesin B.O., Adeniyi J.O., TEC variation within the African EIA region during the recent similar intense geomagnetic storms and IRI-2016 evaluation

Imada S., Iijima H., Hotta H., Kusano K., Shiota D., Predicting solar cycle 25 using surface flux transport model (PSTEP)

Kanekal S., Baker D., Sibeck D., Van Allen probes observations of high energy radiation belt electrons and SEP protons: Space weather aspects

Katsavrias Ch., Daglis I.A., Li W., On the cause of electron acceleration and loss in the outer Van Allen belt

Katsavrias Ch., Sandberg I., Li W., Podladchikova O., Daglis I.A., Papadimitriou C., Tsironis $C_{\text {., }}$ Aminalragia-Giamini $S$. , Highly relativistic electron flux enhancement during the weak geomagnetic storm of April-May 2017

Katsova M.M., The evolution of the solar-stellar activity

Kaushik S.C., Kaushik S., Transient plasma signatures and space weather

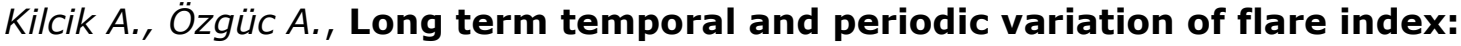
Comparison with international sunspot number time series 
Koleva K., Duchlev P., Dechev M., Miteva R., Hierarchical relations between eruptive prominence properties, flare evolution and CME kinematics in large solar energetic particle events

Kozarev K., Temmer M., Veronig A., Miteva R., Dechev M., Dissauer K., Radial and tangential kinematics of EUV coronal bright fronts: Implications for SEP acceleration efficiency

Krivolutsky A., Cherepanova L., Dement'eva A., Tuniyants T., Climate variability induced by solar cycle (simulations with ARM and data analysis)

Krivolutsky A., Cherepanova L., Vyushkova T., Tuniyants T., Solar cycle in UV and its response in D-region (3D simulations)

Krivova $N$., Understanding and modelling solar and stellar radiative variability

Kukoleva A., Kononova N., Krivolutsky A., Response of circulation indexes in the lower atmosphere of Northern hemisphere to solar cycle

Kusano K., Toward the physics-based prediction of solar storm

Lastovicka J., Progress in long-term trends in the MLT region

Lübken F.-J., ROMIC-2: A future perspective for a new SCOSTEP related science programme in Germany

Mäkelä P., Gopalswamy N., Yashiro S., Akiyama S., Xie H., Michalek G., Data catalogs available at the CDAW data center

Malandraki O.E., Solar energetic particles: Science and applications

Manuilova R., Yankovsky V., Vorobeva Ek., The $\left[\mathrm{CO}_{2}\right]$ altitude profile retrieval from the measured $\mathrm{O}_{2}$ emissions in the mesosphere and lower thermosphere (MLT) region

Martens $P$.C., The faint young Sun paradox mitigated by a sustained initial massive solar wind

Miloch W.J., and IPIR team, Ionospheric plasma irregularities studied globally with the Swarm satellites

Miteva R., Kashapova L., Tsvetkov Ts., Myagkova I., Bogomolov A., Myshyakov I., Petrov N., Zhdanov D., Danov D., The SEP origin project: Results from the Bulgarian-Russian bilateral collaboration

Miteva R., Veronig A., Samwel S.W., Koleva K., Dechev M., Dickson E., Dissauer K., Temmer M., Kozarev K., Kashapova L., On the flare contribution to solar energetic particles

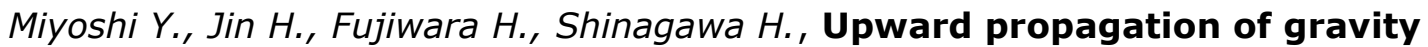
waves and its impact on the thermosphere-ionosphere system

Nade D.P., Potdar S.S., Pawar R.P., Jeni N.V., Nikte S.S., Chavan G.A., Taori A., Siingh $D$., Trend analysis of total column ozone during three recent solar cycles over India

Nandi D., Bhowmik Pr., Century-scale reconstruction of solar activity and a prediction for sunspot cycle $\mathbf{2 5}$

Narayanan V.L., Balan N., A statistical study of co-occurrence of spread $F$ and sporadic $E$ in the middle latitudes

Nasi A., Daglis I.A., Katsavrias Ch., Wen L., Effect of very low frequency (VLF) waves on the acceleration of electrons to relativistic energies 
Nikolova N., Arsov T., Angelov I., Angelov Ch., Participation of BEO Moussala into SEVAN global network

Nosé M., Practice of research data management in solar-terrestrial physics

Obridko V., Ragulskaya M., Khramova E., Young Sun, galactic processes and origin life

Pires R., Fonseca J.M., Mora A., Ribeiro R., Dorotovič I., Rybák J., Automatic detection and tracking of solar features from the AIA/SDO images - a web tool

Podgorny I.M., Podgorny A.I., Mechanism of solar flare based on energy storage in the magnetic field of the current sheet

Popov A.A., Gavrilov N.M., Perminov V.I., Pertsev N.N., Medvedeva I.V., Long-term changes in the intensity of mesoscale variations in hydroxyl rotational temperature near the mesopause as indicators of dynamic processes in the underlying atmosphere

Qian L., Woods T.N., Solar flare effects on the thermosphere and ionosphere

Rabiu B., Okoh D., Shiokawa K., Otsuka Y., Segun B., Falayi E., Preliminary observational investigation of equatorial plasma bubbles over Africa using an all-sky airglow imager and the study of its occurrence frequency

Roussev I.I., Present and future opportunities for geospace science research in the USA

Samwel S.W., Miteva R., Solar energetic electrons and radio emission signatures

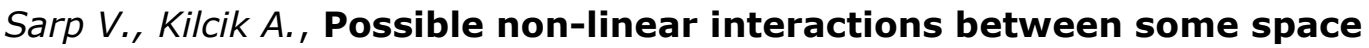
weather parameters

Shen F., Yang Yi, Feng $X$. , Prediction of solar wind parameters at 1 AU using an artificial neural network and multiple observations

Shiokawa K., Otsuka Y., and PWING Team, Recent results of the ground-based multi-point network observation of the upper atmosphere, ionosphere, and magnetosphere by the OMTIs and the PWING project

Shkevov R., Erokhin N.S., Loznikov V.M., Zolnikova N.N., Mikhailovskaya L.A., Predictability of a variable source of high speed streams of ultrarelativistic particles based on the simulation analysis

Suzuki S., Park J., Otsuka Y., Shiokawa K., Liu H., Lühr H., Neutral and plasma density perturbations in the top-/bottom-side ionosphere associated with MSTIDs

Takahashi H., Wrasse C.M., Figueiredo C.A.O.B., Barros D., Abdu M.A., Otsuka Y., Shiokawa $K .$, Paulino $I .$, Equatorial plasma bubble seeding under propagation of MSTIDs and MLT-gravity waves

Temmer M., Holzknecht L., Dumbovic M., Vrsnak B., CME density derivation from remote sensing stereoscopic data

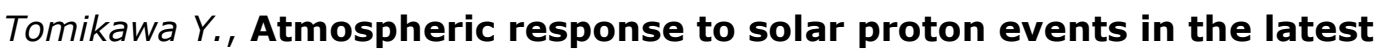
reanalysis data

Török, T., Sun-to-Earth MHD simulations of large solar eruptions

Tsagouri I., Belehaki A., Koutroumbas K., Xenaki I., Quantifying ionospheric disturbances for user oriented applications 
Tsvetkov Ts., Petrov N., Myshyakov I., Miteva R., Solar prominence observations during 2017 total solar eclipse

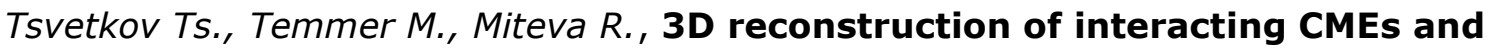
related SEP characteristics

Umuhire A.C., Uwamahoro J., Gopalswamy N., Mungufeni P., Monstein C., Characterization of coronal mass ejections from the associated high frequency type II solar radio bursts

Vashalomidze Z., Zaqarashvili T.V., Kukhianidze V., Prominence eruption triggered by coronal rain in the solar atmosphere observed by SDO/AIA and STEREO/EUVI

Veretenenko S., Ogurtsov M., Temporal variability of solar activity influences on the lower atmosphere and its possible reasons

Veretenenko S., Ogurtsov M., Obridko V., Long-term variations in occurrence frequency of magnetic storms with sudden and gradual commencements

Ward W., Lübken F.-J., Seppala A., Funke B., Krivolutsky A., Woods T., Nakamura T., Stolle Cl., Yigit E., Lastovicka J., Role Of the Sun and the Middle atmosphere/thermosphere/ionosphere In Climate (ROSMIC): A retrospective and prospective view

Watanabe $T$., Data-led study on the Sun-Earth variability and its relationship to socio-economic activity in $18^{\text {th }}-19^{\text {th }}$ centuries including the Dalton minimum

Werner R., Guineva $V_{\text {., }}$ Sunspot number prognoses for the $\mathbf{2 4}^{\text {th }}$ and $\mathbf{2 5}^{\text {th }}$ solar cycle

Wu C.-C., Kan L., Lepping R.P., Solar cycle variation of the heliospheric plasma sheet thickness

Wu C.-C., Kan L., Lepping R.P., Lynn H., The 04-10 September 2017 Sun-Earth connection events: Solar flares, coronal mass ejections/magnetic clouds, and geomagnetic storms

Yashiro S., Gopalswamy N., Akiyama S., Mäkelä P., White-light signatures of shocks driven by coronal mass ejections and interplanetary type II radio bursts

Yermolaev Yu.I., Lodkina I.G., Yermolaev M.Yu., Borodkova N.L., Riazantseva M.O., Properties of interplanetary drivers of magnetospheric disturbances

Yue J., Xu S., Xue X., Vadas Sh.L., Miller S.D., Azeem I., Straka III W., Hoffmann L., Coupling of meteorology severe weather and space weather via the excitations and propagations of gravity waves

Zagainova Yu.S., Fainshtein V.G., Gromova L.I., Gromov S.V., Source regions identification and geophysical effects of stealth coronal mass ejections

Zhang J., Temmer M., Gopalswamy N., ISEST/Minmax24 project: International Study of Earth-affecting Solar Transients 


\section{Sessions}

\section{Mechanisms of solar variability and its Earth-affecting manifestations}

Convener: Dibyendu Nandi, co-conveners: Jacob Bortnik, Jie Zhang, Ilya Usoskin

The Sun is a variable star whose magnetic, radiative and particle output varies over multiple timescales ranging from stellar evolutionary timescales to centennial, decadal and daily timescales; variability across this range of timescales seamlessly couple space climate to space weather. This session will focus on the physical mechanisms that drive solar-stellar variability and create phenomena which eventually impact the Earth and other planets.

\section{Long-term solar variability and its impacts on the heliosphere and} the terrestrial system including solar wind, geomagnetic field, and Earth's climate (Space climate) Convener: Vladimir Obridko, co-conveners: Katya Georgieva, Piet Martens, Kalevi Mursula

Solar activity affects the heliosphere and the terrestrial system on different time scales. In our session, we propose to consider long-term processes with characteristic scales from several years to millions of years. Moreover, activity on the young Sun and its possible influence on the origin of life on Earth will also be discussed. Special attention will be paid to extreme situations (superflares, grand minima). At the same time, the selection of effective and physically justified indices of solar activity becomes important. Finally, the prospects for the upcoming cycle will be discussed.

3. Short-term solar variability and Earth-affecting events, and the reaction of the terrestrial system to solar/heliospheric drivers (Space weather)

Convener: Shri Kanekal, co-conveners: Manuela Temmer, Craig Rodger, William Ward

Short-term solar variability such as coronal mass ejections, high-speed solar wind streams (corotating interaction regions), and interplanetary shocks are the main drivers affecting the terrestrial geospace environment. The space weather effects of these solar transients range from atmospheric to magnetospheric regions surrounding the Earth. This session solicits presentations that address geospace response to these solar drivers from a space weather perspective. Recent missions such as Arase, and Van Allen Probes provide measurements of geospace, interplanetary spacecraft at L1 measure solar driver parameters, both in great detail and of high quality. Both observational as well as model studies that address the space weather impact of short-term solar variability of the geospace system are welcome.

\section{Coupling between the Earth's atmosphere and space under quiet or active Sun}

Convener: Franz-Josef Lübken, co-conveners: Kazuo Shiokawa, Annika Seppälä, Jan Lastovicka

The Earth's atmosphere exhibits a large range of variability which is partly due to intrinsic processes, but also due to forcing from outside, for example by the Sun. At the 
same time, the atmosphere introduces significant variability of the plasma, electromagnetic field, and neutrals in geospace regions. This session focuses on the geospace and middle atmosphere response to the variability of the lower atmosphere, including effects by the propagation of gravity waves, tides, and planetary waves from the lower atmosphere to the thermosphere and ionosphere, as well as their connection to the inner and outer magnetosphere. Presentations on the impact of longer term variability of the atmosphere such as ENSO, QBO, and trends of greenhouse gases are encouraged. Reports on modeling and observational approaches are welcome.

\section{Sun to Earth event case studies Convener: Jie Zhang, co-conveners: David Webb, Yoshi Miyoshi, Franz- Josef Lübken}

This session calls on focused campaign study of Sun-Earth connection events that are of specific significance in science understanding and/or of strong geoeffectiveness. The session fosters inter-disciplinary collaboration in understanding the full chain of activity from Sun to Earth. The known events of interest (arrival at Earth) are 2012/07/14, 2013/03/17, 2013/05/31, 2015/03/17, 2017/09/07 events.

\section{Sun-Earth related data: Definition, maintenance, archiving Convener: Kazuo Shiokawa, co-conveners: Nat Gopalswamy, Katya Georgieva, Aude Chambodut}

A rigorous data management is of upmost importance to allow interoperability between databases, development of tools and interdisciplinary science from the Sun to the Earth. In recent years, projects and research infrastructures have been created to facilitate the access and handling of heterogeneous datasets by scientists. At the same time, International Scientific Instances and Organizations (RDA) are issuing guides and advices on good practices for data life cycle [(i) create, acquire, collect; (ii) check, curate, process, analyze; (iii) ingest, save, preserve; (iv) discover; (v) access, download; (vi) use, cite; (vii) re-use] towards scientific communities, whereas certification of data repositories (ISC-WDS, CoreTrustSeal) is becoming an important concern. Altogether, these initiatives make it possible for users to easily access huge archives of disparate data and metadata in a secure and reliable manner. This session solicits contributions on various data archiving efforts, data repositories mutual links as well as possible related topics as development of visualization tools and plotting software.

\section{Predictability of the Variable Solar-Terrestrial Coupling (PreSTo): The science behind}

Convener: Ioannis A. Daglis, co-conveners: Nat Gopalswamy, Emilia Kilpua, Dan Marsh

PreSTo (Predictability of the variable Solar-Terrestrial Coupling), the Next Scientific Program of SCOSTEP, will address the predictability of the Sun-Earth System on a wide range of timescales. This session will present the overall structure of PreSTo and the scientific orientation of its main pillars. 


\title{
Abstracts
}

\section{Dependence of equatorial ionization gradient with Sq current and F2-layer bottomside thickness parameter in the African region}

\author{
Adebesin B.O., Adeniyi J.O., Adebiyi S.J., Ikubanni S.O. \\ Space Weather Research Group, Department of Physical Sciences, Landmark \\ University, P.M.B 1001, Omu-Aran, Nigeria
}

\begin{abstract}
A first attempt at investigating the dependence of ionization gradient ( $d N / d h)$, solar quiet component $(\mathrm{Sq}(\mathrm{BH}))$, and bottom-side thickness parameter $(\mathrm{BO})$ in the African equatorial sector. Digisonde and MAGDAS data collocated at Ilorin, an equatorial station was employed. Result was presented for sunrise midday, sunset, and midnight local hours. The ionization gradient peak height remains unchanged at midday across all months. A percentage correlation of $93 \%$ existed in the inverse and direct linear relationship of $\mathrm{dN} / \mathrm{dh}-\mathrm{BO}$ at sunrise, and of $\mathrm{Sq}(\mathrm{BH})-\mathrm{B} 0$ at midday respectively. Significant relationship between $\mathrm{dN} / \mathrm{dh}$ and $\mathrm{Sq}(\mathrm{BH})$ was at sunset; for other hours, the relationship is poor. The multiple linear relationship of $\mathrm{dN} / \mathrm{dh}$ $\mathrm{Sq}(\mathrm{BH})-\mathrm{BO}$ parameters revealed that the dependence of $\mathrm{dN} / \mathrm{dh}$ on $\mathrm{Sq}(\mathrm{BH})$ and $\mathrm{BO}$ is highest at midday, and a model equation was presented. The dependence of $\mathrm{dN} / \mathrm{dh}$ on the solar activity index (F10.7) holds at all the selected hours, and distinct only at midday and midnight for the $\mathrm{Sq}(\mathrm{BH})-\mathrm{F} 10.7$ and $\mathrm{B} 0-\mathrm{F} 10.7$ patterns. The importance of the vertical plasma drift and F-region dynamo at heights above $150 \mathrm{~km}$ was established.
\end{abstract}

\section{Pattern of digisonde measured-scale height derived at an African equatorial station}

Adebiyi S.J., Adeniyi J.O., Adebesin B.O., Ikubanni S.O.

Department of Physics, Space Weather group, Landmark University, Omu-Aran, Nigeria

For topside density profile extrapolation, one important parameter used in topside profilers is the scale height which can be estimated from ionogram. For the first time in the African equatorial region, the variation of digisonde derived scale height $(\mathrm{Hm})$ is investigated using data from Ilorin (Geog. $8.50^{\circ} \mathrm{N} ; 4.50^{\circ} \mathrm{E} ;$ dip. $-4.1^{\circ}$ ). The diurnal variation shows an early morning increase with maximum around the noon period, followed by a decrease till nighttime. The variation however shows significant seasonal trend. A notable finding is the highest value observed in December solstice and the lowest in June solstice over the station. This is attributed to the influence of the ion-neutral drag and the topside thermal structure on the shape of the topside ionosphere. $\mathrm{Hm}$ also has a good correlation with the $\mathrm{F} 2$ layer bottomside thickness parameter (B0) and the total electron content (TEC); and moderate correlation with F2-layer critical frequency (foF2) and peak height (hmF2). The correlation coefficient obtained however do not show significant seasonal difference. The study also demonstrates that the good correlation between $\mathrm{H} \_\mathrm{m}$ and $\mathrm{BO}$ could provide an excellent opportunity to determine $\mathrm{H} \_\mathrm{m}$ from the already established BO model (if well modeled at the station) for possible application in topside profilers. 


\title{
Geoeffectiveness of the interplanetary coronal mass ejections over the years 1996-2017
}

\author{
Alexakis P., Mavromichalaki H. \\ National and Kapodistrian University of Athens, Greece
}

The interplanetary coronal mass ejections (ICMEs) being the Interplanetary manifestations of the coronal mass ejections, are considered as important drivers of intense geomagnetic activity. This characteristic makes them vital parameters for Space Weather Forecasting, indicating that it is crucial for the forecasters to study their geoeffectiveness. In this work, the distributions and the main plasma parameters of about 500 ICMEs that are their velocities, the southward component of their magnetic field Bs and their convective dawn-to-dusk electric field Ey are examined for the last two solar cycles (1996-2017). To what extent they affected the Earth's magnetosphere and what are the probabilities of reaching specific levels of geomagnetic storms are also studied. For this purpose these ICME parameters with their geoeffective signature depending on two main geomagnetic indices, $\mathrm{Kp}$ and Dst, are correlated with interesting results. The differences of these ICME characteristics and their geoeffectiveness during the two solar cycles 23 and 24, are investigated in detail and discussed.

\section{The long-lasting QP emissions observed on ERG satellite and Lovozero station}

\author{
Belakhovsky V.B. ${ }^{1}$, Shiokawa K. ${ }^{2}$, Matsuoka A. ${ }^{3,4}$, Kasahara Y.5, Shinohara I. ${ }^{3,4}$, \\ Miyoshi $Y^{2}$ \\ 1 Polar Geophysical Institute, Apatity, Russia \\ 2 Institute for Space-Earth Environmental Research, Nagoya, Japan \\ ${ }^{3}$ Institute of Space and Astronautical Science, Japan \\ 4 Japan Aerospace Exploration Agency, Japan \\ ${ }^{5}$ Kanazawa University, Japan
}

In this work the simultaneous QP emission registered on ERG satellite and Lovozero station (Kola Peninsula) of Polar Geophysical Institute are investigated. We select only those events when the ERG satellite and Lovozero station was geomagnetically conjugate. The simultaneous OP emission on the ground and in space was observed during about 70 minutes in the evening sector of MLT. There is very high correlation between QP elements on the ground and in space. The QP emissions was registered in a frequency range $1-2 \mathrm{kHz}$, the period of QP emissions was approximately 40 seconds. Inside the QP emissions the higher frequency fine structures were observed. It is interesting that according to the ERG satellite data QP emissions are observed only in electric field, in magnetic field QP emissions are not observed.

It is found that QP emissions are not accompanied by the geomagnetic pulsations with the same period on the ground-based magnetometers. But according to the magnetometer data of ERG satellite with using spectral analysis it is identified the frequency very close to the frequency of QP emissions. The appearance of these QP emissions can be associated with the small-scale Pc3 pulsations which seen in space and does not seen on the ground due to its damping in the ionosphere. So these QP emissions should be attributed to the QP1 class. Thus even if we don't see geomagnetic pulsations on the ground it does not necessarily indicate that this is QP2 class of QP emissions. 


\title{
The generation of monochromatic PC4 pulsations by the electron cloud
}

Belakhovsky V.B. ${ }^{1}$, Shiokawa K. ${ }^{2}$, Matsuoka A. ${ }^{3,4}$, Wang S.-Y.5, Kazama Y. ${ }^{5}$, Tam S. ${ }^{6}$, Kasahara S. ${ }^{7}$, Yokota S. ${ }^{8}$, Keika K. ${ }^{7}$, Hori T. ${ }^{2}$, Shinohara I. ${ }^{3,4}$, Miyoshi Y. ${ }^{2}$

1 Polar Geophysical Institute, Apatity, Russia

2 Institute for Space-Earth Environmental Research, Nagoya, Japan

${ }^{3}$ Institute of Space and Astronautical Science, Japan

4 Japan Aerospace Exploration Agency, Japan

${ }^{5}$ Academia Sinica Institute of Astronomy and Astrophysics, Taiwan

${ }^{6}$ National Cheng Kung University, Taiwan

7 Tokyo University, Japan

8 Osaka University, Japan

In this work we investigate the physical nature and generation mechanism of very monochromatic Pc4 pulsations with using data of ERG (Exploration of energization and Radiation in Geospace) satellite. Such type of the pulsations is exited during very low geomagnetic activity, these pulsations are do not seen on the ground magnetometers due to damping in the ionosphere. The question about the generation mechanism of these pulsations is still open.

For the event 4 May 2017 according to the ERG satellite data the wave packet of Pc4 pulsations was registered after midnight at 08-10 UT. The pulsations are mostly seen in radial component and also in azimuthal component of the magnetic field; its frequency is about $13 \mathrm{mHz}$. On GOES-13 satellite located on higher L-shell than ERG satellite the frequency of these pulsations is about $11.5 \mathrm{mHz}$. This property (decrease of the frequency of geomagnetic pulsations with the increase of L-shell) testifies about the resonance nature of the pulsations.

During appearance of the Pc4 pulsations on ERG satellite the injection of electrons (mostly seen in 10-80 keV energetic cannels) are registered. This injection is coincide with the small increase of $A E$ index up $200 \mathrm{nT}$. So this injection is caused by the small substorm. Thus it is found the experimental evidence that injection of electron cloud into the morning sector can be reason of the excitation of monochromatic Pc4 geomagnetic pulsations. There are some theories which can explain this mechanism.

\section{Real-time identification of Travelling Ionospheric Disturbances and validation methodologies}

\author{
Belehaki A. ${ }^{1}$, and TechTIDE EC consortium ${ }^{2}$ \\ ${ }^{1}$ National Observatory of Athens, Greece \\ 2 TechTIDE Horizon 2020, European Commission Project 776011
}

Travelling Ionospheric Disturbances (TIDs) are an important Space Weather effect in the upper atmosphere constituting a threat for operational systems using predictable ionospheric characteristics. TIDs can impose disturbances with amplitudes of up to $\sim 20 \%$ of the ambient electron density, and a Doppler frequency shifts of the order of $0.5 \mathrm{~Hz}$ on HF signals.

The TechTIDE-HORIZON 2020 project aims at delivering in real-time data, products and services capable of detecting the occurrence of TIDs over specific regions. Furthermore, in close collaboration with operators of the technologies concerned, TechTIDE consortium will design and test new viable TID impact 
mitigation strategies and will demonstrate the added value of the proposed mitigation techniques which are based on TechTIDE products.

In this contribution we present first results from the TID detection methodologies that are based on the analysis of Digisonde, GNSS and Doppler Sounding data using complementary modeling techniques for Medium Scale and Large Scale TID identification. More precisely we apply the methodologies based on the analysis of Digisonde to Digisonde bistatic operations and Doppler Soundings, on HF Interferometry, on Spatial and Temporal analysis of GNSS measurements, on TEC gradients, and on the results from topside electron density profile reconstruction models.

The analysis focuses primarily on periods of enhanced auroral activity. TechTIDE identifies perturbations in the amplitude of the electron density and in the HF propagation characteristics (azimuth, elevation, Doppler shift). The validation plan is based on the assessment of the results from all complementary methodologies in respect to geospace and the lower atmosphere conditions.

\title{
Using CUDA supercomputing for acceleration of calculations in study of solar flare mechanism for coronal MHD simulation
}

\author{
Borisenko A.V. ${ }^{1}$, Podgorny I.M. ${ }^{2}$, Podgorny A.I. ${ }^{1}$ \\ ${ }^{1}$ Lebedev Physical Institute RAS, Moscow, Russia \\ 2 Institute of Astronomy RAS, Moscow, Russia
}

Comparison of observations with results of MHD simulation above the active region NOAA 10365 performed in reduced time scale unequivocally is showed, that the flare takes place in corona as a result of release of energy, which is accumulated in the magnetic field of the current sheet. For the first time, a current-sheet model for explaining the mechanism of a solar flare was proposed in 1966 by SI Syrovatsky. IM Podgorny and AI Podgorny performed MHD simulation of formation and evolution of the current sheet above the active region. Basing on the mechanism of flare energy release in a current sheet I.M. Podgorny proposed electrodynamical model of the solar flare which explains main manifestations of a flare. For more detail study of the current sheet magnetic field configuration it is necessary to perform MHD simulation in real time scale. Simulation in real time during the first few minutes of evolution showed the absence of instability at the boundary, which leads to generation of parasitic currents. Simulations must be carried out so that, in the absence of instability at the photospheric boundary, it is possible to more conveniently and more accurately determine the position of the current sheet. For this purpose, the "personal supercomputer" on the base of CUDA NVIDIA technology with the GPU Tesla M2050 has been installed. Hardware and software were installed and tested. We are currently prepare MHD simulation above a multi-flare large complex active region NOAA 12192 to determine current sheets in the corona.

\section{The evolving solar-stellar dynamo}

\author{
Brun A.S., Strugarek A.
}

CEA Paris-Saclay, Département d'Astrophysique, France

In this talk we will discuss how a solar-like star builds up its magnetic field via dynamo action and how much of its luminosity is needed to maintain this nonlinear turbulent process. We will make a detailed analysis of energy fluxes within stellar 
convection zones and how differential rotation and magnetic energies are being maintained. By varying the stellar parameters we will derive various scaling laws that will help us understand how solar like stars magnetism evolve on short to long (secular) time scales.

\title{
Reconciling the sunspot and group number series
}

Clette F., Lefèvre $L$.

\author{
Royal Observatory of Belgium, Brussels, Belgium
}

The release of the first revision of the sunspot number (SN) series in 2015 was motivated by the abnormally large discrepancies between this time series and its closest counterpart, the sunspot group number (GN), which persisted since the publication of the GN series in 1998.

We describe first the very different construction of the two series, and the totally different corrections that were derived independently for the SN and GN series. The two resulting series have now come in a much closer agreement, indicating that artificial defects affecting each series were effectively eliminated. While the validity of the new version (V2.0) of the SN series is confirmed by various comparisons, several incompatible versions of the GN series have been published since 2015 . We introduce the different approaches leading to those GN series, and the ongoing assessment of the underlying methods (daisy chaining of $\mathrm{k}$ personal coefficients, 'backbone' reference observers, active-day fraction). This joint evaluation in the framework of international workshops (ISSI, Bern) has revealed methodological limitations and flaws, which we describe.

We finally introduce the completely new approach adopted to prepare the upcoming SN Version 3: construction of a complete digital archive of historical observations, which will feed a full re-construction of the SN series from all raw source data. We conclude on the non-linear relation between the SN and GN series, which after reconciliation, i.e. elimination of past processing defects, could provide a long-term probe of solar cycle properties over past centuries.

\section{State of the art and gaps on the role of interplanetary structures on Sun-Earth coupling}

\author{
Dasso $S$.
}

\section{CONICET - Universidad de Buenos Aires, Instituto de Astronomía y Física del Espacio (IAFE), Buenos Aires, Argentina}

Universidad de Buenos Aires, Facultad de Ciencias Exactas y Naturales, Departamento de Ciencias de la Atmósfera y los Océanos and Departamento de Física, Buenos Aires, Argentina

From several decades ago it is well stablished that interplanetary properties play a crucial role on the Sun-Earth coupling. However, even when during last year's new significant knowledge about solar origin and evolution of these interplanetary structures have been learned, some questions are still open.

In the frame of SCOSTEP's Next Scientific Program (NSP), and under the general concept of the "Predictability of the variable Solar-Terrestrial System" (PreSTo), a 
committee worked on receiving feedback on open scientific issues and needs of the solar-terrestrial community.

In this invited talk I will briefly present main aspects of the state of the art about interplanetary drivers for Sun-Earth connection, and will present the identified gaps in our solar-terrestrial coupling understanding and the ways to achieve progress in the NSP areas according with the work developed by this committee.

\title{
First observations from the space weather Antarctic node of the Latin American Giant Observatory (LAGO)
}

\author{
Dasso S. ${ }^{1,2,3}$, Gulisano A.M. ${ }^{3,4}$, Areso 0. ${ }^{1}$, Pereira M. ${ }^{1}$, et al. ${ }^{5,6}$, \\ for the LAGO collaboration ${ }^{7}$ \\ ${ }^{1}$ IAFE/UBA CONICET, Argentina \\ 2 UBA FCEyN, Departamento de Ciencias de la Atmósfera y los Océanos (DCAO), \\ Argentina \\ 3 UBA FCEyN, Departamento de Física (DF), Argentina \\ ${ }^{4}$ Instituto Antártico Argentino/DNA, Argentina \\ ${ }^{5}$ Servicio Meteorológico Nacional (SMN), Argentina \\ ${ }^{6}$ Centro Atómico Bariloche (CNEA/CONICET/IB, Instituto de Tecnologías en Detección y \\ Astropartículas (CNEA, CONICET, UNSAM), Argentina \\ 7 http://lagoproject.net
}

We will present cosmic rays fluxes observed by a water Cherenkov radiation detector, the first Antarctic node of the LAGO collaboration (Latin American Giant Observatory) located in the Antarctic Space Weather Laboratory of the LAMP group (Laboratorio Argentino de Meteorología del esPacio). This laboratory and the LAGO node, installed in the Marambio base during January-February of 2019, are located at 64.24S-56.62W, and 200 meters above sea level. The laboratory also has a meteorological station measuring atmospheric pressure, external/internal temperatures, and humedity.

The rigidity cut off due to the presence of the geomagnetic field is lower at higher latitudes. Thus, the Antarctic Continent has the unique advantage of combining a territory with infrastructure for the location of astroparticle detectors, and it allows the arrival of cosmic rays with lowest energies to ground level. These particles are tracers that carry a large amount of information, linked with physical processes in the Sun and the interplanetary space.

We will present the activities developed during the installation of the Cherenkov detector at the Argentine Marambio Base of the Antarctic Peninsula, along with the creation of the Space Weather laboratory.

We will also present the data repositories and system developed for providing the measurements from this detector on a real-time base for operative Space Weather aims.

\section{Case study of a filament eruption associated with a C8.4 flare}

\author{
Dechev M., Duchlev P., Koleva K.
}

Institute of Astronomy and NAO, Bulgarian Academy of Sciences, Bulgaria

We study a filament eruption in NOAA ARS: 11562 S16E42, 11563 S25E57. We observed two eruptions: first - at about 17:00UT; second - after 20:00 UT. The 
filament seems to have completely erupted and a C8.4 flare was registered. Some filament materials are seen to come back, at AIA/SDO $304 \AA$. The compact active region has become part of the flare ribbon. The filament material demonstrates a lot of twisted, dynamic structures. We measured the kinematic parameters of the erupted material, twist angle and discuss the possibility of some instabilities to occur.

\title{
External drivers of decadal variations in the geomagnetic field and Earth's rotation
}

\author{
Demetrescu Cr. ${ }^{1}$, Dobrica V. ${ }^{1}$, Georgieva K. ${ }^{2}$, Kirov B. ${ }^{2}$ \\ ${ }^{1}$ Institute of Geodynamics, Romanian Academy, Romania \\ 2 Space Research and Technology Institute - Bulgarian Academy of Sciences, Bulgaria
}

We compare oscillations seen in the geomagnetic field at the solar-cycle timescale to variations at the same timescale we show to be present in the Earth's rotation rate and in the variations of the magnetospheric ring current. We isolate solar-cycle variations using the Hodrick and Prescott (1977) procedure that provides a cyclic component, which we identify as the solar-cycle-related variation, and a long-term trend. The link between the geomagnetic field and Earth's rotation at century timescales has long been accepted in general as a manifestation of the Earth's angular momentum conservation (e.g. Le Mouël et al., 1981). We advance the hypothesis of possible external drivers of variations observed in declination $(\mathrm{dD} / \mathrm{dt})$ and length of the day (LOD) as oscillations generated either by variations in the magnetospheric ring current, via induction in the upper part of the outer core, or by a direct control of declination by variations in the ring current.

\section{Energetic particles in the heliosphere and their impacts on geospace}

\author{
Desai M.I., Dayeh M.A., Ebert R.W. \\ Southwest Research Institute, University of Texas at San Antonio, USA
}

Coronal mass ejections and solar flares release huge quantities of energized matter, magnetic fields and electromagnetic radiation into space. The high-energy particles, known as solar energetic particles or SEPs, present a serious radiation threat to human explorers living and working outside low-Earth orbit and to technological assets such as communications and scientific satellites in space. Our current understanding of SEPs is hampered because the near-Earth spacecraft observations are influenced by a combination of multiple effects such as the identity of the suprathermal seed populations, the mechanisms by which these seed particles are selected and accelerated to higher energies, and lastly, how interplanetary turbulence and large-scale solar wind structures affect their transport to Earth orbit. Our limited understanding also affects the abilities of current CME and SEP acceleration models to predict the occurrence, intensity, and impacts of the accompanying radiation storms in the near-Earth environment. This talk discusses key areas in which missions like the Parker Solar Probe (PSP), Solar Orbiter, and the Interstellar Mapping and Acceleration Probe (IMAP) will provide ground-truth observations that will serve as definitive tests for current SEP models. 


\title{
Space weather event on 7-8 September 2017: Magnetic supersubstorms and dayside polar substorms
}

\author{
Despirak I.V. ${ }^{1}$, Kleimenova N.G. ${ }^{2}$, Gromova L.I. ${ }^{3}$, Gromov S.V. ${ }^{3}$, Malysheva L.M. ${ }^{2}$ \\ 1 Polar Geophysical Institute, Apatity, Russia \\ 2 Schmidt Institute of Physics of the Earth, RAS, Moscow, Russia \\ ${ }^{3}$ IZMIRAN, RAS, Moscow, Troitsk, Russia
}

The severe $(\mathrm{Kp}=8)$ geomagnetic storms on 7 and 8 September 2017 were associated with two consecutive large scale solar wind structures: SHEATH with EJECTA (on 7 September) and SHEATH with magnetic cloud - MC (on 8 September). The positive IMF Bz on the front edge of the first SHEATH resulted an absence of the substorm activity in this time. Later on, when IMF Bz became negative, the strong (up to $800 \mathrm{nT}$ ) night-side substorms appeared simultaneously with dayside polar substorms. When the second SHEATH arrived with the strong negative IMF Bz (up to $-30 \mathrm{nT}$ ), the main phase of the first magnetic storm began, and the night-side supersubstorm (up to $3500 \mathrm{nT}$ ) developed accompanying by the weaker (up to $1000 \mathrm{nT}$ ) dayside polar magnetic bays. In the first storm recovery phase, under positive IMF $\mathrm{Bz}$, the magnetic activity was concentrated in the dayside polar region. The MC impact under the negative IMF Bz caused the second magnetic storm and the severe night-side supersubstorm (up to $~ 3500 \mathrm{nT}$ ) and the dayside polar magnetic bay-like disturbances. Thus, during the 7-8 September 2017 storms, two supersubstorms were generated. We suppose that dayside polar substorms, observed simultaneously with the supersubstorms, were not typical dayside bays associated with NBZ FAC, but they were the result of the westward ionospheric current extension toward the dayside. Thus, the storm-time superstorms caused by the SHEATH and MC impact have demonstrated the global scale distribution.

\section{THEMIS and ground-based observations during the event on 24 December 2014}

\author{
Despirak I.V., Kozelova T.V., Kozelov B.V., Lubchich A.A. \\ Polar Geophysical Institute, Apatity, Russia
}

The analysis of the substorm activity on December 24, 2014 was carried. During 19-27 December 2014 several solar wind structures (shock waves, magnetic cloud, high speed stream etc.) were observed. As a result, two geomagnetic storms, on 21 and 23 December, were developed. We analyzed of substorm, which was observed during the recovery phase of the second storm, in the interval $\sim 16: 10-\sim 17: 30$ UT. During this period, the substorm disturbances of the fields and fluxes of energetic particles were recorded on THE and THD satellites (at $r \sim 8.5-10.3 \mathrm{Re}$ ). The disturbances in the geomagnetic field are registered at Tiksi (TIK), Dickson (DIX), Amderma (AMD), Lovozero (LOZ) stations and IMAGE network. Aurora activity was observed by all-sky camera in Apatity (APT). It is shown, that the active arc associated with the "onset" of this substorm was located on the latitude, between THD and THE, because the different signs of the EX components on the two satellites were observed near moment T0. It's confirmed the almost simultaneous beginning of the growth of the magnetic field and plasma fluxes in both satellites but with a slight advance on the more eastward satellite (THE). This idea is supported the appearance of a discrete, localized on latitude, auroral arc (so-called "auroral 'horn') at the all-sky camera in Apatity. 


\title{
Advances and limitations in physics-based predictions of short- term and decadal solar activity
}

\author{
Dikpati M.
}

\author{
NCAR, High Altitude Observatory, USA
}

\begin{abstract}
Polar field precursor technique has been one of the important methods for predicting next solar cycle's strength for many years. Recently, after the success of this technique in forecasting cycle 24 amplitude, there is now wide agreement that polar field from previous cycle is a good predictor for next sunspot cycle's amplitude. Despite the validation of cycle 24 overall strength prediction, this technique fails to predict cycle amplitudes in North and South hemispheres separately, as well as the North-South asymmetry in cycle-phase. So an obvious remaining question is: do we really understand the physical connection between polar fields and sunspotproducing toroidal fields? Dynamo models show that two hemispheres operate nearly independently, implying weak hemispheric-coupling. Some dynamo ingredients are not known from observations, and are also difficult to constrain from theory, so it might seem that we are prevented from making improvements in dynamo-based predictions. However, there exists substantial solar data for differential rotation, meridional circulation as well as surface magnetograms which have not yet been used systematically in dynamo simulations. I will discuss how the inclusion of data assimilation techniques can provide improvements. Equally important is predicting short-term or 'seasonal' (6-18 months) solar activity bursts, because most major space weather events originate from these bursts, and occur even in weak cycles. For example, a major Carrington type event occurred in cycle 24, the weakest in 100 years. I will close by discussing the recent advances in predicting seasonal activity bursts and their implication in decadal cycle predictions.
\end{abstract}

\section{Surface electric field over Europe as hazardous response to the intense/severe geomagnetic storms of the solar cycle 24}

\author{
Dobrica V., Demetrescu Cr., Greculeasa R., Stefan Cr. \\ Institute of Geodynamics, Romanian Academy, Romania
}

The variable magnetic field produced by the interaction of the solar wind and heliospheric magnetic field with the magnetosphere and ionosphere induces hazardous electric currents in ground technological systems, the so-called geomagnetically induced currents (GICs). To assess ground space weather impact of GICs we need information on the induced surface electric field. In this study we determine the surface electric field over Europe produced by the variable magnetic field of geomagnetic storms, based on the geomagnetic field recordings from European geomagnetic observatories and on information regarding the underground electric conductivity. The geographical distribution of the maximum induced surface geoelectric field over Europe by the intense/severe geomagnetic storms of the solar cycle 24 is presented as well. 


\title{
Eruptions of two coupled flux ropes of a filament and associated ribbon flare and CME
}

\author{
Duchlev P., Dechev M., Koleva K.
}

Institute of Astronomy and National Astronomical Observatory, Bulgarian Academy of Sciences, Bulgaria

We present a study of a filament eruption on 2015 July 19 that observed close to the limb of southwest hemisphere. The filament, composed of two coupled main flux ropes (FRs), underwent activation between 00:00 UT and 04:00 UT. After the filament eruption, two coupled main FRs present different behavior, as observed in AIA/SDO 304 A channel. Top dark FR rise more rapidly than bottom bright FR. At 08:55 UT bottom FR reaches top FR and during next 15 minutes the FRs rise almost coherently. Afterward, top dark FR begins accelerates while the bottom FR continues slowly rise. At 10:00 UT, when top FR is high in the corona, bottom FR begins accelerates and escaped the AIA field-of-view at 10:40 UT. This specific filament eruption produces a pair of long flare ribbons, which became a gradual C2.1 flare. It was also associated with a decent coronal mass ejection (CME). Here we determined the kinematic parameters of the FRs, their twist angles and discuss the possible instabilities of eruption. We find the causal relationships between the filament FRs eruptions, ribbon flare, CME kinematics and associated SEP event.

\section{Investigations on upper atmospheric variability on a global scale due to the influence of solar forcing from above and vertical coupling from below}

\author{
Duggirala P. ${ }^{1}$, Singh R.P. ${ }^{1}$, Mandal S. ${ }^{1}$, Keran D.K. ${ }^{1}$, Laskar F.I. ${ }^{2}$ \\ ${ }^{1}$ Physical Research Laboratory, Navrangpura, Ahmedabad, India \\ 2 Leibniz Institute of Atmospheric Physics, Kuhlungsborn, Germany
}

Upper atmospheric regions are sandwiched due to solar forcing from above and from below in terms of waves. Forcing from below can be due to tropical storms (effect on the upper atmosphere is of the order of one/two days), stratospheric sudden warming (SSW) (order of couple of weeks), planetary scale waves (order of several days), seasonal (order of months), and much larger timescales (order of solar activity). In the past decade systematic and concerted investigations on upper atmospheric dynamics using airglow emissions, during both day and night times, and ionospheric measurements from low-latitudes yielded exciting new insights such as, vertical coupling of atmospheres being dependent on the phase of the solar activity, upper atmospheric gravity wave activity showing a solar activity dependence, setting up of new meridional circulation during SSW, a double-humped structure with respect to geographic equator in mesospheric temperatures, existence of longitudinal differences in equatorial processes in as small as 3 degree separation, gravity wave propagation being different during different geomagnetic disturbances, and a commonality in the source of high-latitude total electron content and ring current variations. Several of these results demonstrate the intricate role played by the variation in the solar activity and space weather in defining the ionospherethermosphere-mesosphere dynamics. These variabilities are required to be incorporated into global scale models for an improved description of the ionospherethermosphere system. Some of the results, especially those during SSW periods, and on the efficacy of vertical coupling during solar activity will be presented. 


\title{
Utilizing galactic cosmic rays to understand the Sun-to-Earth evolution of CMEs
}

\author{
Dumbovic M.1,2 \\ ${ }^{1}$ Institute of Physics, University of Graz, Austria \\ 2 Hvar Observatory, University of Zagreb, Croatia
}

Traditionally, to identify ICMEs in the in-situ measurements, mostly plasma and magnetic field measurements are regarded. However, one of the very common insitu signatures of ICMEs, as well as other interplanetary transients are Forbush decreases (FDs), i.e. short-term reductions in the galactic cosmic ray (GCR) flux. FD phenomena are caused by the interaction of GCRs with a magnetic structure, therefore it is expected that different types of interplanetary substructures cause different types of GCR depressions, allowing us to distinguish between shock/sheath, flux rope and SIR-type of FDs. Moreover, since the interaction of GCRs and CME magnetic structure (presumably flux rope) occurs all the way from Sun to Earth, FDs should also reflect the evolutionary properties of CMEs, which is supported by the results from recent modeling efforts (Dumbovic et al., 2018, ApJ). The evolutionary process of different magnetic structures in interplanetary space will be discussed in the scope of selected case studies, utilizing a number of multi-instrument and multispacecraft observations, together with modelling. This research has received funding from the European Union's Horizon 2020 research and innovation programme under the Marie Skłodowska-Curie grant agreement No 745782.

\section{Tilted equatorial plasma bubble and plasma blobs after interaction with medium-scale traveling ionospheric disturbances and midnight brightness wave}

\author{
Figueiredo C.A.O.B. ${ }^{1}$, Takahashi H. ${ }^{1}$, Wrasse C.M. ${ }^{1}$, Otsuka Y. ${ }^{2}$, Shiokawa K. ${ }^{2}$, \\ Buriti R.A. ${ }^{3}$, Paulino I. ${ }^{3}$, Barros D. ${ }^{1}$ \\ ${ }^{1}$ Instituto Nacional de Pesquisas Espaciais, São José dos Campos, Brazil \\ 2 Institute for Space-Earth Environmental Research, Nagoya University, Nagoya, Japan \\ ${ }^{3}$ Department of Physics, Universidade Federal de Campina Grande, Campina Grande, \\ Brazil
}

We report simultaneous observation of equatorial plasma bubbles (EPBs), medium-scale traveling ionospheric disturbance (MSTID), and midnight brightness wave (MBW) in the emission of the OI $630 \mathrm{~nm}$ obtained by All-Sky imager located at Cachoeira Paulista, Brazil $\left(22.4^{\circ} \mathrm{S}, 45^{\circ} \mathrm{W}\right.$, magnetic dip latitude $\sim 20^{\circ} \mathrm{S}$, referring to 2015) on the night of September 17-18, 2015. While the EPBs were moving eastward, a dark band type MSTID propagated northwestward with the wave front aligned to southwest-northeast. Around the local midnight, the MBW moved to south-southwest. When the MSTID interacted with the EPBs, the EPBs were bifurcated, elongated and tilted; we associate this effect to the ExB drift of MSTID. Furthermore, a MBW interacts with EPB and fills the EPB edge with MBW plasma transforming in plasma blobs. 


\title{
Towards a better understanding of EPP effects on the middle atmosphere and climate
}

Funke $B$.

Instituto de Astrofísica de Andalucía, CSIC, Granada, Spain

Precipitating energetic particles from the Sun and the magnetosphere affect the ionization levels in the polar middle and upper atmosphere, leading to significant changes of the chemical composition. In particular, the production of NOx and HOx imposes changes of ozone via catalytic cycles, potentially affecting temperature and winds. Vertical coupling of this signal to the lower atmosphere could provide a link between space weather in the form of energetic particle precipitation (EPP) and surface climate: model studies and the analysis of meteorological data have indeed provided evidence for EPP-induced climate variations on the regional scale. This talk summarizes recent progress on this topic. Further, open issues, obstacles and requirements for advancing our knowledge of EPP impacts on the middle atmosphere and potential climate links are discussed.

\section{Dynamical and thermal effects of nonlinear acoustic-gravity waves in the upper atmosphere at high and low activity}

\author{
Gavrilov N.M. ${ }^{1}$, Kshevetskii S.P. ${ }^{2}$ \\ ${ }^{1}$ Saint-Petersburg State University, Atmospheric Physics Department, St. Petersburg, \\ Russia \\ ${ }^{2}$ Immanuel Kant Baltic Federal University, Theoretical Physics Department, \\ Kaliningrad, Russia
}

Majority of acoustic-gravity waves (AGWs) observed at high altitudes are supposed to be originated in the lower atmosphere by different sources and propagate upwards. A high-resolution three-dimensional numerical model describing vertical propagation of nonlinear AGWs to the upper atmosphere from the ground was recently developed. Numerical modeling was made in a region of the Earth atmosphere with dimensions $500 \mathrm{~km}$ vertically and up to several thousand kilometers horizontally. Vertical profiles of the mean density, temperature, thermal conductivity and molecular viscosity are taken from standard atmospheric models. Numerical modeling for background vertical temperature and wind profiles matching high and small levels of solar activity (SA) shows that AGWs characteristics in the middle and upper atmosphere may change with changes in SA. The wave amplitudes at altitudes above $150 \mathrm{~km}$ are larger at high SA because of smaller wave-induced mean wind and smaller molecular heat conduction and viscosity. Wave accelerations of the mean flow are generally larger at low SA. This produces faster grows of wave-induced jet streams in time at low SA. Velocities of the wave-induced mean flows are higher at Iow SA compared to high SA. Resulting SA impact depends on competition between AGW amplitude increase due to smaller molecular dissipation and smaller energy transfer to the wind-induced mean flow and amplitude decrease caused by larger density and stronger reflection at higher SA. Acknowledgements. This study was supported by the Russian Basic Research Foundation \#17-05-00458. 


\title{
Data management, analysis tools and science applications by the Plasma Physics Data Center (CDPP)
}

\author{
Génot V., and the CDPP team
}

CNRS, UPS, CNES, Observatoire de Paris, France

20 years after its creation the CDPP gathers a variety of plasma data from ionosphere, heliophysics and planetology experiments and has extended its expertise to analysis tools and virtual observatory concepts. This presentation will show how by specializing thematic software but making them interoperable it is possible cover most of space scientist usages from data access, visualisation, and mining. The CDPP 'eco-system of tools ' will be presented together with recent applications (machine learning, 3D simulation rendering, ...).

\section{Solar energetic particle event associated with the 2017 September 6 coronal mass ejection}

\author{
Gopalswamy N. ${ }^{1}$, Akiyama S. ${ }^{2}$, Mäkelä P. ${ }^{2,1}$, Yashiro S. ${ }^{2,1}$, Xie H. ${ }^{1}$ \\ 1 NASA Goddard Space Flight Center, Greenbelt, MD 20771, USA \\ 2 Catholic University of America, Washington DC 20064, USA
}

A series of three large solar energetic particle events occurred during September 4-10, 2017. Of these the SEP event on September 6 was also associated with an intense geomagnetic storm with a peak intensity of $-124 \mathrm{nT}$. In addition to a detailed description of the source region of the September 6 eruption that can be related to the geomagnetic storm, we provide a comparative analysis of the three SEP events. We use the Flux Rope from Eruption Data (FRED) technique to relate the source magnetic properties to the ICME properties. We also report on the fluence spectra of the three SEP events and how they can explain the occurrence of sustained gammaray emission (SGRE) during the last two events (Gopalswamy et al. 2018, ApJL 868, L19).

\section{A catalog of interplanetary type II radio bursts observed by the Wind/WAVES instrument}

\author{
Gopalswamy N. ${ }^{1}$, Mäkelä P. ${ }^{2,1}$, Yashiro S. ${ }^{2,1}$, Akiyama S. ${ }^{2}$ \\ ${ }^{1}$ NASA Goddard Space Flight Center, Greenbelt, MD 20771, USA \\ ${ }^{2}$ Catholic University of America, Washington DC 20064, USA
}

The Radio and Plasma Wave Experiment (WAVES) on board the Wind spacecraft has been providing information on low-frequency radio emission from the Sun since its launch in 1994. A catalog of these type II bursts observed in the decameterhectometric $(\mathrm{DH})$ to kilometric $(\mathrm{km})$ wavelengths is maintained at NASA's CDAW Data Center (https://cdaw.gsfc.nasa.gov/CME_list/radio/waves_type2.html). For each type II burst in the list, the associated flare and coronal mass ejection (CME) are identified and their properties listed. Links to Javascript movies that combine white-light images from SOHO coronagraphs and Wind/WAVES dynamic spectra and GOES light curves are provided. Links are also provided to the height-time plots of 
the CMEs and plots that combine flare light curve, CME height-time history, and SEP flux. The CME images and WAVES dynamic spectra have the option to make measurements using point and click method. In addition to describing the catalog, we summarize the properties of CMEs and type II bursts over the past two solar cycles.

\title{
Predicting the solar-cycle strength from the polar brightness: Contribution from the Nobeyama Radioheliograph
}

\author{
Gopalswamy N. ${ }^{1}$, Yashiro S. ${ }^{2,1}$, Mäkelä P. ${ }^{2,1}$, Akiyama S. ${ }^{2}$ \\ 1 NASA Goddard Space Flight Center, Greenbelt, MD 20771, USA \\ 2 Catholic University of America, Washington DC 20064, USA
}

The polar field strength inferred from the Nobeyama Radioheliograph's $17 \mathrm{GHz}$ brightness temperature can be used for solar-cycle prediction. The polar microwave brightness temperature is highly correlated with the polar magnetic field strength and the fast solar wind speed. The polar microwave brightness in one solar cycle is correlated with the low latitude brightness with a lag of about half a solar cycle. We use this correlation to show that solar cycle 25 will not be too different from cycle 24 in its strength. We also combine the rush-to-the-pole data from Nobeyama prominences with historical data going back to 1860 to show that the north-south asymmetry of sign reversal at solar poles has a quasi-periodicity of 3-5 cycles.

\section{Substorm activity at high and middle latitudes during large geomagnetic storms}

\author{
Guineva V. ${ }^{1}$, Despirak I.V. ${ }^{2}$, Kleimenova $N .{ }^{3}$, Werner R. $^{1}$ \\ ${ }^{1}$ Space Research and Technology Institute - BAS, Stara Zagora Department, Bulgaria \\ 2 Polar Geophysical Institute, Apatity, Murmansk region, Russia \\ ${ }^{3}$ Schmidt Institute of the Earth Physics RAS, Moscow, Russia
}

The spatial-temporal dynamics of magnetic substorms during the initial and main phases of large geomagnetic storms (SYM/Hmin < -200 nT) has been studied. The presence and spreading of substorms were examined on the basis of the INTERMAGNET and IMAGE networks data. The concomitant solar wind and Interplanetary Magnetic Field parameters were taken from the OMNI data base. A comparative analysis of the interplanetary conditions during substorms which propagated to middle and low latitudes, and during substorms which didn't extend below auroral latitudes, under similar level of geomagnetic activity was performed. The conditions favorable for the development of "classical" and "expanded" substorms were also investigated. We suggested that the occurrence of such kind of substorms is related to the different solar wind conditions: the "classical" substorms developed under magnetic cloud (MC) conditions, and the "expanded" - under the Sheath region influence. 


\title{
Effect of solar variability on the solar wind during cycle 23 and
} 24

\author{
Hazra S., Brun A.S., Buchlin E., Reville V., Strugarek A., Perri B. \\ Laboratoire AIM, DSM/IRFU/SAp, CEA Saclay, F-91191 Gif-sur-Yvette Cedex, \\ France
}

\begin{abstract}
Stellar wind is generally believed as a responsible mechanism for the spin down of main-sequence stars. Many studies has been performed on the extraction of angular momentum by stellar winds. However, most of the previous studies generally consider simple dipole or quadrapolar magnetic topologies. In this work, we consider realistic magnetic topologies of the Sun, observed at the Wilcox Solar Observatory. We perform simulations at different phases of the solar cycle with a 2.5D spherical, axisymmetric solar wind model, with realistic magnetic topology as a bottom boundary condition. We are able to reproduce general solar wind structure and recover the solar like values of mass loss rate and angular momentum loss. Finally, we compare our polytropic wind solution to solutions including a more realistic heating source term based on Alfven wave turbulence.
\end{abstract}

\section{The predictability of the Earth's radiation belts}

Horne R.B.

\author{
British Antarctic Survey, UK
}

Over the last five years or more we have made substantial progress in understanding the Earth's radiation belts. New data from satellites such as RBSP and ARASE have highlighted the importance of magnetopause compression, radial diffusion and resonant wave-particle interactions for controlling the variability of the radiation belts. We have also learned that there are almost no electrons with energies greater than $800 \mathrm{keV}$ in the inner belt, and that fast solar wind streams can cause some of the highest fluxes in the outer belt. As space weather becomes more important for the thousands of new satellites that are being launched here we examine our ability to predict the radiation belts. We examine the type of events where prediction needs to be improved, and this in turn helps to set a new focus on the problems that need to be addressed. These range from our ability to forecast Sun to Earth, the amount of electron loss into the atmosphere and its effects on the atmosphere, to the cause of flux drop-out events, the role of quasi-linear and nonlinear theory, and what we need to understand in order to forecast extreme events.

\section{TEC variation within the African EIA region during the recent similar intense geomagnetic storms and IRI-2016 evaluation}

\author{
Ikubanni S.O., Adebiyi S.J., Adebesin B.O., Adeniyi J.O. \\ Space Weather Research Group, Department of Physical Sciences, Landmark \\ University, Omu-Aran, Nigeria
}

The 2015 event is the most intense of earth-directed solar storms in the 24th solar cycle. Another intense event in 2013 with same driving factors, occurring on 
identical season and time during similar solar activity epoch provides a good opportunity to study the geospace response to geomagnetic disturbance. In this presentation, the response of Global Positioning System (GPS)-derived Total Electron Content (TEC) at some African stations, particularly in the equatorial ionization anomaly (EIA) region, will be analyzed and discussed. The analysis covers from the storm day to about 72 hours after the main phase. The performance of the 2016 version of the International Reference Ionosphere (IRI) in reproducing the storminduced variations in TEC is also evaluated. IRI could not capture the effect of the electrodynamics on the ionosphere due to input to additional energy into the ionosphere.

\title{
Predicting solar cycle 25 using surface flux transport model (PSTEP)
}

\author{
Imada S., Iijima H., Hotta H., Kusano K., Shiota D. \\ Nagoya Univ., Chiba Univ., NICT, Japan
}

It is thought that the longer-term variations of the solar activity may affect the Earth's climate. Therefore, predicting the next solar cycle is crucial for the forecast of the "solar-terrestrial environment". To build prediction schemes for the next solar cycle is a key for the long-term space weather study. Recently, the relationship between polar magnetic field at the solar minimum and next solar activity is intensively discussed. Because we can determine the polar magnetic field at the solar minimum roughly 3 years before the next solar maximum, we may discuss the next solar cycle 3years before. Further, the longer term ( $\sim 5$ years) prediction might be achieved by estimating the polar magnetic field with the Surface Flux Transport (SFT) model. Now, we are developing a prediction scheme by SFT model as a part of the PSTEP (Project for Solar-Terrestrial Environment Prediction) and adapting to the Cycle 25 prediction. We find that the observed axial dipole moment becomes approximately constant during the period of several years before each cycle minimum, which we call the axial dipole moment plateau. These results allow us to predict the axial dipole moment at the Cycle 24/25 minimum using the SFT model without introducing new flux emergence. The predicted axial dipole moment at the Cycle 24/25 minimum is $60-80$ percent of Cycle 23/24 minimum, which suggests the amplitude of Cycle 25 is even weaker than the current Cycle 24.

\section{Van Allen probes observations of high energy radiation belt electrons and SEP protons: Space weather aspects}

\author{
Kanekal S., Baker D., Sibeck D. \\ NASA GSFC, LASP University of Colorado, NASA GSFC, USA
}

Relativistic and ultra-relativistic electrons as well as solar energetic particles (SEP) pose a space weather hazard both to spacecraft and humans in space. Coronal mass ejections, high speed streams and IP shock interaction with magnetosphere cause energization and enhanced levels of relativistic electrons which remain trapped in the Earth's radiation belts for long periods (weeks to months). IP shocks are also responsible SEP energization in the interplanetary space. SEPs reach near Earth regions via open field lines over the polar caps and are also at times observed to 
penetrate into the inner magnetosphere. We review observations from Van Allen Probes since its launch in fall 2012. Van Allen Probes mission comprises two identical spacecraft in a near equatorial orbit, each carrying a comprehensive suite of particles and fields instruments. Here, we focus mainly upon energetic particle observations, viz., electrons and protons, from the REPT (Relativistic Electron Proton Telescope) instrument. REPT measure electrons from about 2 to $20 \mathrm{MeV}$ and protons from about 18 to $100 \mathrm{MeV}$ in pitch angle resolved multiple differential channels. We provide an overview of energetic particle events observed by Van Allen Probes since launch to present and discuss potential space weather aspects of energetic particle radiation.

\title{
On the cause of electron acceleration and loss in the outer Van Allen belt
}

\author{
Katsavrias Ch. ${ }^{1,2,3}$, Daglis I.A. ${ }^{1,2,3}$, Li W. $^{4}$ \\ ${ }^{1}$ Department of Physics, National and Kapodistrian University of Athens, Athens, \\ Greece \\ ${ }^{2}$ Institute of Accelerating Systems and Applications, Athens, Greece \\ ${ }^{3}$ Institute for Astronomy, Astrophysics, Space Applications and Remote Sensing, \\ National Observatory of Athens, Penteli, Greece \\ ${ }^{4}$ Center for Space Physics, Boston University, Boston, USA
}

We investigate the response of the outer Van Allen belt electrons to various types of solar wind and internal magnetospheric forcing, to geospace magnetic storms of different intensities and to intense magnetospheric substorms. We use electron phase space density (PSD) calculations as well as concurrent Pc5 and chorus wave activity observations in the outer belt during the Van Allen Probes era to compare 20 electron enhancement and 8 depletion events. Our results indicate that the combined effect of magnetopause shadowing and outward diffusion driven by Pc5 waves is present in both groups of events. Furthermore, in the case of enhancement events, the synergy of enhanced seed population levels and chorus activity - due to the enhanced substorm activity - can effectively replenish the losses of relativistic electrons while, inward diffusion can further accelerate them.

\section{Highly relativistic electron flux enhancement during the weak geomagnetic storm of April-May 2017}

\author{
Katsavrias Ch. ${ }^{1,2}$, Sandberg I. ${ }^{3}$, Li W. ${ }^{4}$, Podladchikova O. ${ }^{5}$, Daglis I.A. ${ }^{1,2,6}$, \\ Papadimitriou C. ${ }^{3}$, Tsironis C. ${ }^{2,7}$, Aminalragia-Giamini S. ${ }^{3}$ \\ ${ }^{1}$ National and Kapodistrian University of Athens, Greece \\ 2 Institute of Accelerating Systems and Applications, National and Kapodistrian \\ University of Athens, Greece \\ ${ }^{3}$ Space Applications and Research Consultancy (SPARC), Athens, Greece \\ ${ }^{4}$ Center for Space Physics, Boston University, USA \\ ${ }^{5}$ Solar-Terrestrial center of excellence, Royal Observatory of Belgium, Belgium \\ ${ }^{6}$ Institute of Astronomy, Astrophysics, Space Applications and Remote Sensing, \\ National Observatory of Athens, Greece \\ 7 School of Electrical and Computer Engineering, National Technical University of \\ Athens, 15773 Athens, Greece
}

We report observations of energetic electron flux and Phase Space Density (PSD) to show that a relatively weak magnetic storm, with SYM-H reaching only $-50 \mathrm{nT}$, 
resulted in a relativistic and ultra-relativistic electron enhancement of two orders of magnitude similar to the St. Patrick's event of 2015, an extreme storm with SYM-H reaching $-235 \mathrm{nT}$. This enhancement appeared at energies up to $\approx 10 \mathrm{MeV}$, lasted for at least 24 days and was not recorded in geosynchronous orbit where most space weather alert data are collected. By combined analysis of PSD radial profiles and Fokker-Planck simulation, we show that the enhancement of relativistic and ultrarelativistic electrons is caused by different mechanisms: first, chorus waves during the intense substorm injections of April 21-25 accelerate the seed electron population to relativistic energies and redistribute them while inward diffusion driven by Pc5 ULF waves further accelerates them to ultra-relativistic energies.

\title{
The evolution of the solar-stellar activity
}

\author{
Katsova M.M.
}

Sternberg State Astronomical Institute, Lomonosov Moscow State University, Russia

I review observational results focused on the solar-stellar connections, which help us to trace changes in physical conditions on the Sun at different stages of its life. Estimate of stellar ages from the period of the axial rotation (the method of the gyrochronology) permits us to study dependence of the activity on the age. The quasi-stationary X-Ray- and FUV-radiation of the outer atmosphere of the young Sun is estimated. Both most powerful non-stationary phenomena and quasi-stationary activity are discussed on the base of space missions and ground-based observations. Joint analysis of superflares and stellar magnetic fields on solar-type stars gives a chance to estimate the maximal possible energy of stellar flares; that allows us to understand the origin of extreme events on the Sun.

\section{Transient plasma signatures and space weather}

\author{
Kaushik S.C. ${ }^{1}$, Kaushik S. ${ }^{2}$ \\ ${ }^{1}$ Department of Physics, Government PG Autonomous College (Jiwaji University), \\ Datia, India \\ 2 School of Physics, Jiwaji University, Gwalior, India
}

One of the challenge for space weather research today is to quantitatively predict the dynamics of the geo-magnetosphere from observed solar wind and interplanetary magnetic field (IMF) conditions. In the present study a correlative study between geomagnetic storms and the various interplanetary (IP) field / plasma parameters have been performed to search the perpetrators of geomagnetic activity and to develop such model suitable for predicting the occurrence of geomagnetic activities, significant for space weather predictions. We have investigated solar wind and IMF parameters in different situations and also derived the linear relationship for all parameters in different situations based on the peak values of Disturbance storm time index (Dst). These results inferred that the southward Bz component of the interplanetary magnetic field is an important factor for describing geomagnetic storms however its magnitude is not found maximum neither during the initial phase of the storm, i.e. at the instant of the interplanetary shock nor during the main phase, the instant of minimum Dst index. It is also investigated that there is a time delay between the maximum value of southward Bz and the Dst minimum, and this time delay can be used in the prediction of the intensity of a magnetic storm two- 
three hours before the main phase of a geomagnetic storm. We have found some more factors influencing the initiation of storm like, the speed of the solar wind, plasma beta should be low and, most importantly, the plasma temperature should be low enough for intense storms.

\title{
Long term temporal and periodic variation of flare index: Comparison with international sunspot number time series
}

\author{
Kilcik A. ${ }^{1}$, Özgüc A. ${ }^{2}$ \\ ${ }^{1}$ Akdeniz University, Faculty of Science, Department of Space Science and \\ Technologies, 07058, Antalya, Turkey \\ ${ }^{2}$ Kandilli Observatory and Earthquake Research Institute, Bogazici University, 34684 \\ Istanbul, Turkey
}

Here, we used Ha flare index (FI) data taken for the time period of 1966-2018 (total 19360 days), which covers from Cycle 20 to 23 completely and almost whole cycle 24 (2009-2018). This data set compared with similar activity index International Sunspot Number (ISSN) data that arise under different physical conditions during the studied time period. Then temporal and periodic variations of hemispheric and whole data were analyzed by using the cross-correlation analysis method, Morlet wavelet and MTM period analysis methods. It is found that; i) both north and south hemisphere FI data show the same level of correlation $(r=0.71)$ which is lower than the total FI data $(r=0.82)$, with ISSN, ii) these hemispheric FI data sets also show some cyclic differences in periodicity analysis.

\section{Hierarchical relations between eruptive prominence properties, flare evolution and CME kinematics in large solar energetic particle events}

\author{
Koleva K. ${ }^{1}$, Duchlev P. ${ }^{1}$, Dechev M. ${ }^{1}$, Miteva $R .^{2}$ \\ ${ }^{1}$ Institute of Astronomy and National Astronomical Observatory, Bulgarian Academy of \\ Sciences, Sofia, Bulgaria \\ 2 Space Research and Technology Institute, Bulgarian Academy of Sciences, Sofia, \\ Bulgaria
}

We report a detailed study of the relationship between solar filament eruption, flare productivity and CME kinematics relevant to Solar Energetic Particle (SEP) events. We analyze kinematics and morphological behaviors of 29 filament eruptions appearing during the Solar Cycle 24, between 14 Aug 2010 and 18 April 2016. For our analysis a high resolution data in different Extreme Ultraviolet (EUV) channels from Solar Dynamic Observatory (SDO) are used. Each of the observed eruptions is associated with solar flare, observed in hard X-rays (HXRs) and is followed by in situ observed proton events. The kinematics properties of the associated CME are tracked for the observed events. We perform a statistical chronological study of the observed sequence of events in order to reveal the eruption triggers and the evolved physical mechanisms. 


\title{
Radial and tangential kinematics of EUV coronal bright fronts: Implications for SEP acceleration efficiency
}

\author{
Kozarev K. ${ }^{1}$, Temmer M. ${ }^{2}$, Veronig A. ${ }^{2}$, Miteva $R .^{3}$, Dechev M. ${ }^{1}$, Dissauer K. ${ }^{2}$ \\ ${ }^{1}$ Institute of Astronomy and National Astronomical Observatory - Bulgarian Academy \\ of Sciences, Sofia, Bulgaria \\ 2 Institute of Physics-IGAM, University of Graz, Graz, Austria \\ ${ }^{3}$ Space Research and Technology Institute-Bulgarian Academy of Sciences, Sofia, \\ Bulgaria
}

Large-scale solar coronal compressive waves, or coronal bright fronts (CBFs) are often observed in extreme UV (EUV) during nascent coronal mass ejections (CMEs), which develop most dynamically (expansion, acceleration) in the low and middle solar corona (below 5-8 solar radii). Multiple studies have found a connection between CBFs and driven coronal shock waves, and their ability to accelerate coronal ions to solar energetic particle (SEP) energies. A commonly invoked condition for the generation of EUV waves and their capability to produce energetic particles is the presence of rapid lateral expansion of the front and driver behind it. We present a study of the radial and lateral kinematics of a number of CBFs from SDO/AIA observations, and the later-stage CME kinematics from SOHO/LASCO observations. We investigate the nominal particle acceleration efficiency of the CBFs with the CASHeW framework.

\section{Climate variability induced by solar cycle (simulations with ARM and data analysis)}

\author{
Krivolutsky A., Cherepanova L., Dement'eva A., Tuniyants T.
}

Central Aerological Observatory, Dolgoprudny, Moscow Region, Russia

GCM ARM (Atmospheric Research Model [Krivolutsky et al., 2015]) was used to simulate global response of atmospheric temperature and wind to variability in solar UV radiation. Upper boundary of ARM is at $135 \mathrm{~km}$. Global fields of radiative-active gases were incorporated from

3D global photochemical model CHARM [Krivolutsky et al., 2015]. Planetary waves global structure at lower boundary with zonal wave numbers $S=1,2,3$ has been incorporated in the model on the basis of observations. The results of simulations showed that temperature response has wave-like structure with its amplitudes, which are more than several $\mathrm{K}$ in the stratosphere and troposphere. So, revealed response has several minima and maxima along the latitude. Using reanalysis data the similar to wave-like structure was found. So, it was shown, that solar cycle manifestation may has positive or negative sign at the same time at different areas.

\section{Solar cycle in UV and its response in D-region (3D simulations)}

Krivolutsky A., Cherepanova L., Vyushkova T., Tuniyants T.

Central Aerological Observatory, Dolgoprudny, Moscow Region, Russia

Three-dimensional photochemical model CHARM-I (Chemical Atmospheric Research Model with Ions [Krivolutsky et al., 2015]) was used to study changes in 
D-region induced by solar UV variations between minima (2009) and maxima (2003) of solar cycle. Wind components and temperature fields has been calculated with Middle atmosphere Lower Thermosphere GCM ARM (Atmospheric Research Model [Krivolutsky et al., 2015]). Selected interval belongs to 23rd solar cycle with a deep minima in 2007-2009. The results of simulations showed that corresponding changes in electron density for day time conditions equaled about $30 \%$ with minus (in comparison with 2003) above $70 \mathrm{~km}$. At the same time a weak increase of [e] was found in zonal mean fields around $60 \mathrm{~km}$. It seems that this effect depends on layered structure of ozone and atomic oxygen response. Found changes in electron density may be important for lower frequency radio wave propagation.

\title{
Understanding and modelling solar and stellar radiative variability
}

\author{
Krivova $N$.
}

\author{
Max Planck Institute for Solar System Research, Germany
}

Over four decades of space-borne solar irradiance measurements have shown that solar radiative output varies on all observable time scales. Good understanding of this variability has been gained and models have been developed that reproduce the measured variations with high accuracy. A large body of stellar photometric data delivered by recent missions such as CoRoT, Kepler or TESS reveal similar variability for other Sun-like stars, although with a much wider variability patters. I will discuss the state-of-the-art in modelling of the solar irradiance variability, recent efforts to extend this knowledge to understand and model the photometric variability of Sunlike stars, and how this knowledge can, in turn, help us to understand solar variability.

\section{Response of circulation indexes in the lower atmosphere of Northern hemisphere to solar cycle}

\author{
Kukoleva A., Kononova N., Krivolutsky A. \\ Central Aerological Observatory, Dolgoprudny, Moscow Region, Russia
}

The results of the analysis of the relationship of circulation characteristics in the lower atmosphere of the Northern hemisphere with the cycle of solar activity are presented. For the analysis used data on the duration of different types of elementary circulation processes (classification Dzerdzeevskii) and time series of wolf numbers for the period 1899-2016. Applied statistical analysis the method of 'superimposed epochs", the elements of correlation and spectral analysis. It was found that the increase in solar activity in General contributes to the increase in the duration of meridional circulation forms, but the effect on different subtypes of elementary circulation mechanisms is different. Seasonal differences in the effect of the Sun on the lower atmosphere were also found. 


\title{
Toward the physics-based prediction of solar storm
}

Kusano K.

\author{
ISEE, Nagoya University, Japan
}

\begin{abstract}
Severe space weather disaster caused by the large-scale solar storm is a potential risk for modern society. However, because the solar-terrestrial dynamics is governed by complex nonlinear processes, we could not yet understand what determines the occurrence and properties of large-scale space weather events. Therefore, although the prediction is the only way to mitigate the influence of space weather disaster, the predictive scheme currently in operation largely relies on empirical law, and the applicability to the extreme events is severely restricted. To improve our understanding and predictability of the solar-terrestrial dynamics, we should challenge the physics-based prediction of the solar dynamics and the influence on the terrestrial environment. In this paper, for making the next scientific program of SCOSTEP, we discuss the current status about the predictability of solar storm and the prospect of the physics-based prediction through the experience of Project for Solar-Terrestrial Environment Prediction (PSTEP) which is the nation-wide space weather/climate project of Japan.
\end{abstract}

\section{Progress in long-term trends in the MLT region}

Lastovicka J.

Institute of Atmospheric Physics CAS, Prague, Czech Republic

Working group 3 of ROSMIC VarSITI has been dealing with long-term trends in the mesosphere and lower thermosphere (MLT region). Even though the carbon dioxide is the main trend driver in MLT, there are also other trend drivers like ozone, water vapor, change sin atmospheric wave activity, or maybe also secular change of the main magnetic field of the Earth. This could make the trends temporally and spatially unstable. Some important findings in observational trends in the MLT region will be treated in this paper particularly from the point of view of their consistency with the global scenario of long-term trends in the upper atmosphere-ionosphere system.

\section{ROMIC-2: A future perspective for a new SCOSTEP related science programme in Germany}

\author{
Lübken F.-J. \\ Leibniz-Institute of Atmospheric Physics, Schloss-Str. 6, 18225 Kuehlungsborn, \\ Germany
}

The German Federal Ministry of Education and Research (Bundesministerium für Bildung und Forschung, BMBF) launched a research initiative in 2013/2014 called ROMIC (Role of the Middle Atmosphere in Climate). This program terminated in 2017. Based on the success of ROMIC the BMBF announced a second phase in October 2017 which concentrates on two main topics: A) How does the variability of the Sun induce climate changes via processes such as general circulation, chemical 
composition of the middle atmosphere, and other physical processes; B) How do processes in the troposphere, in particular anthropogenic changes, couple to the middle atmosphere, and how do related changes feedback to tropospheric climate change. Many proposals were submitted to BMBF and a selection processes based on external reviews took place in autumn 2018. The selected proposals were invited to submit their final proposals until 28. February 2019. Assuming that all these proposals are finally accepted, a total of 8 cooperative projects with several subprojects will be engaged in ROMIC-2. A total of 14 research institutes in Germany will be involved which cover a large range of experimental and theoretical Topics relevant for ROMIC-2. The scientific goals of these Projects will be presented in more detail.

\title{
Data catalogs available at the CDAW data center
}

\author{
Mäkelä P. ${ }^{1,2}$, Gopalswamy N. ${ }^{2}$, Yashiro S. ${ }^{1,2}$, Akiyama S. ${ }^{1,2}$, Xie H. ${ }^{1,2}$, Michalek G. ${ }^{3}$ \\ 1 The Catholic University of America, Washington DC, USA \\ 2 NASA Goddard Space Flight Center, Greenbelt, USA \\ ${ }^{3}$ Astronomical Observatory of Jagiellonian University, Krakow, Poland
}

The CDAW Data Center has been established and developed to support Coordinated Data Analysis Workshops (CDAWs), to maintain and update solareruption-related databases and make them available to the scientific community online. The currently available set of CDAW databases consist of event lists of all coronal mass ejections (CMEs) observed by SOHO/LASCO coronagraphs, halo CMEs, decameter-hectometric type II radio bursts observed by Wind/WAVES radio experiment, large solar energetic particle events observed by GOES, and automatically-detected SDO prominence eruptions. The CDAW Data Center is widely used by scientific community and, as of June 2018, 1620 refereed papers have been published using these databases. The CDAW Data Center also has online tools, which enable users to make measurements using spacecraft data available on the site. We will review the contents and usage of the databases available at the CDAW Data Center.

\section{Solar energetic particles: Science and applications}

\author{
Malandraki O.E.
}

National Observatory of Athens, IAASARS, GR-15236 Penteli, Greece

Solar Energetic Particles (SEPs) constitute an important contributor to the characterization of the space environment. They are emitted from the Sun in association with solar flares and Coronal Mass ejection (CME)-driven shock waves. SEP radiation storms may have durations from a period of hours to days or even weeks and have a large range of energy spectrum profiles. These events pose a threat to modern technology strongly relying on spacecraft and are a serious radiation hazard to humans in space, and are additionally of concern for avionics and commercial aviation in extreme circumstances. The main objective of the Working Group on SEPS (WG6) of the VarSITI/ISEST project, directly aligned with the ISEST science objectives, has been the improvement of our understanding of the origin, acceleration and transport of energetic particles in the heliosphere, in association with CMEs and Corotating Interaction Regions (CIRs) propagation and evolution. In 
this talk, an overview of the main results and the progress made within WG6 will be reported.

\title{
The $\left[\mathrm{CO}_{2}\right]$ altitude profile retrieval from the measured $\mathrm{O}_{2}$ emissions in the mesosphere and lower thermosphere (MLT) region
}

\author{
Manuilova R., Yankovsky V., Vorobeva Ek. \\ Saint-Petersburg State University, Atmospheric Physics Department, Saint-Petersburg, \\ Russia
}

In the framework of the YM2011 model of electronic-vibrational kinetics, we have demonstrated that collisional quenching with $\mathrm{CO} 2$ is essential for the only one proxy, $\mathrm{O} 2(\mathrm{~b} 1, \mathrm{v}=0)$ [1]. The remaining proxies, $\mathrm{O}(1 \mathrm{D}), \mathrm{O} 2(\mathrm{~b} 1, \mathrm{v}=2$ or 1$), \mathrm{O} 2(\mathrm{a} 1, \mathrm{v}=0$ or 1), do not depend on [CO2] as it was shown using the photochemical model YM2011. However, the population of the level O2(b1, v = 0) depends also on the overlying level $\mathrm{O} 2(\mathrm{~b} 1, \mathrm{~V}=1)$ due to the fast $\mathrm{E}-\mathrm{V}$ energy transfer at collissional quenching. Thus, to retrieve the altitude profile of [CO2] in the daytime MLT, we developed a method that uses the simultaneous measurement of the volume emission rates for the transitions from two excited levels of the oxygen molecule, $\mathrm{O} 2(\mathrm{~b} 1, \mathrm{v}=0)$ and $\mathrm{O} 2(\mathrm{~b} 1, \mathrm{v}=1)$. The uncertainty of retrieval of the $\mathrm{CO} 2$ concentration was estimated by the Monte Carlo method taking into account the errors of all the YM2011 model parameters. The proposed algorithm allows to obtain the altitude profile of $\mathrm{CO} 2$ concentration in the range of $50-85 \mathrm{~km}$ with an uncertainty of $10-20 \%$ and in the range of $85-100 \mathrm{~km}$ with an uncertainty of 20$60 \%$. We compared the results obtained by the Monte Carlo method with the results of the sensitivity analysis [1]. Funding: This work was supported by the Russian Fund for Basic Research (grant RFBR No.17-05-00532).

\section{The faint young Sun paradox mitigated by a sustained initial massive solar wind}

\author{
Martens P.C. \\ Dept. of Physics \& Astronomy, Georgia State University, Atlanta, Georgia, USA
}

It has been demonstrated that the torque required to slow down the young Sun from a rotation period of the order of five days to its current rotation rate requires a sustained ( $3 \mathrm{Gyr}$ ) massive solar wind, or an unrealistically large lever arm for the Alfven surface, well beyond the orbit of Venus (Martens, 2017). A sustained massive solar wind would resolve the well-known 'Faint Young Sun Paradox', in that a young Sun more massive by several percent than the current Sun would be sufficiently more luminous to prevent the young Earth from turning into a permanent 'Snowball Earth ' - for which there is no geological and paleo-climatological evidence. There is some observational indication for such a massive solar wind, sustained for $3 \mathrm{Gyr}$ (Melendez et al, 2017). I am performing standard solar evolution calculations for two scenarios. The first is the standard one in which an initial massive mass loss of the order of $1 \% / G y r$ rapidly declines to the current rate. The second scenario is one in which the same mass loss is sustained for about three billion years, which, as mentioned above, can explain both the spin-down rate typical for solar-type stars 
and resolve the Faint Young Sun paradox. I will discuss what the observable differences are between the two scenarios for the current Sun with regard to composition, luminosity, rotation rate, etc.

\title{
Ionospheric plasma irregularities studied globally with the Swarm satellites
}

\author{
Miloch W.J. ${ }^{1}$, and IPIR team ${ }^{1,2}$ \\ 1 Department of Physics, University of Oslo, Oslo, Norway \\ 2 Helmholtz Centre Potsdam GFZ German Research Centre for Geosciences, Potsdam, \\ Germany
}

The Earth's ionosphere is often subject to instabilities and turbulence, which can lead to irregularities in the plasma density at various scales and geomagnetic latitudes. The ionospheric plasma density irregularities depend on the geomagnetic activity. They can influence the propagation of radio signals, which can degrade the communication services or precise positioning with the Global Navigation Satellite Systems (GNSS), such as GPS, Galileo or GLONASS. As such, ionospheric plasma irregularities are an important component of the space weather system.

In this work we characterize plasma density irregularities in the ionosphere at all latitudes using data from the Swarm satellites. By employing the Swarm IPIR dataset, including the IPIR index, we assign irregularities to different geomagnetic regions, and provide their typical properties under different geomagnetic conditions. We then focus on the low- and high-latitude phenomena, including polar cap patches and equatorial bubbles, and discuss the corresponding variations in the plasma density and the topside total electron content as measured by Swarm. A brief introduction to the IPIR data product is also given.

\section{The SEP origin project: Results from the Bulgarian-Russian bilateral collaboration}

\author{
Miteva R. ${ }^{1}$, Kashapova L. ${ }^{2}$, Tsvetkov Ts. ${ }^{3}$, Myagkova I. ${ }^{4}$, Bogomolov A. ${ }^{4}$, \\ Myshyakov I. ${ }^{2}$, Petrov N. ${ }^{3}$, Zhdanov D. ${ }^{2}$, Danov D. ${ }^{1}$
}

${ }^{1}$ Space Research and Technology Institute, Bulgarian Academy of Sciences, Bulgaria

${ }^{2}$ Institute of Solar Terrestrial Physics - SB Russian Academy of Sciences, Russia

${ }^{3}$ Institute of Astronomy and National Astronomical Observatory, Bulgarian Academy of Sciences, Bulgaria

${ }^{4}$ Skobeltsyn Institute of Nuclear Physics, Lomonosov Moscow State University, Russia

We present results of a two-year bilateral project between Bulgarian and Russian teams on the origin of solar energetic particles (SEPs). The project results are organized according to the topics of the five work packages, namely SEPS, SEPrelated flares, SEP-related coronal mass ejections, the SEP-origin identification, and dissemination. A three-language web-page hosts the project science topic and information about the team, results and public outreach materials: http://newserver.stil.bas.bg/SEPorigin/. We summarize the main project achievements and present recommendations for future studies. This study is cofunded by the National Science Fund of Bulgaria under contract No. DNTS/Russia 01/6 (23-Jun-2017) and the Russian Foundation for Basic Research with project No. 17-52-18050. 


\title{
On the flare contribution to solar energetic particles
}

Miteva R. ${ }^{1}$, Veronig A. ${ }^{2}$, Samwel S.W. ${ }^{3}$, Koleva K. ${ }^{1}$, Dechev M. ${ }^{4}$, Dickson E. ${ }^{2}$, Dissauer K. ${ }^{2}$, Temmer M. ${ }^{2}$, Kozarev K. ${ }^{4}$, Kashapova L. ${ }^{5}$

${ }^{1}$ Space Research and Technology Institute, Bulgarian Academy of Sciences, Bulgaria 2 IGAM, Institute of Physics, University of Graz, Austria

${ }^{3}$ National Research Institute of Astronomy and Geophysics, Helwan, Cairo, Egypt

${ }^{4}$ Institute of Astronomy and National Astronomical Observatory, Bulgarian Academy of Sciences, Bulgaria

${ }^{5}$ Institute of Solar Terrestrial Physics - SB Russian Academy of Sciences, Russia

The solar flare emission in relationship to solar energetic particles (SEPs) is investigated in the present study. Extreme ultraviolet (EUV), hard X-ray (HXR) and microwave radio (MW) wavelengths are selected and analyzed. Different time periods are considered in each case according to the data coverage provided by the specific instrument. Namely, SDO/EVE data is from 2011 to 2014, RHESSI HXR data - in selected observing windows during the period 2002-2018 and RSTN MW data covers 1996-2018. The peak SEP intensities from both, SOHO/ERNE $20 \mathrm{MeV}$ protons and ACE/EPAM 175-315 keV electrons are considered. Pearson correlations are calculated between the various flare emission and SEP amplitudes. The outcomes are compared with the corresponding result when coronal mass election projected speed is considered instead of the flares. This study is co-funded by the National Science Fund of Bulgaria under contracts No. NTS/Austria 01/23 (28.01.2017) and DNTS/Russia 01/6 (23-Jun-2017), Austria OeAD Project No. BG 11/2017 and the Russian Foundation for Basic Research with project No. 17-52-18050.

\section{Upward propagation of gravity waves and its impact on the thermosphere-ionosphere system}

\author{
Miyoshi ${ }^{1}{ }^{1}$, Jin $H_{.}{ }^{2}$, Fujiwara $H .{ }^{3}$, Shinagawa $H^{2}$ \\ ${ }^{1}$ Kyushu University, Japan \\ 2 National Institute of Information and Communications Technology, Japan \\ 3 Seikei University, Japan
}

It has been recognized that gravity waves (GWs) play an important role on the momentum and energy budget in the thermosphere/ionosphere. In this study, using a whole atmosphere-ionosphere coupled model (GAIA) with high horizontal resolution, behaviors of GW in the mesosphere and thermosphere and their impacts on variability in the ionosphere. GAIA contains the region from the ground surface to the upper thermosphere, so that we can simulate excitation of GWs in the lower atmosphere, their upward propagation from the lower atmosphere to the upper atmosphere, and their impacts on the thermosphere-ionosphere system. GAIA can simulate traveling ionospheric disturbances TIDs, because interaction processes between the ionosphere and neutral atmosphere are incorporated. We focus our attention on TIDs generated by upward propagating GWs during geomagnetically quiet condition. Vertical coupling processes between the lower and upper atmospheres by GWs are discussed. 


\title{
Trend analysis of total column ozone during three recent solar cycles over India
}

\author{
Nade D.P. ${ }^{1,2}$, Potdar S.S. ${ }^{2}$, Pawar R.P. ${ }^{1}$, Jeni N.V. ${ }^{3}$, Nikte S.S. ${ }^{4}$, Chavan G.A. ${ }^{5}$, \\ Taori A. ${ }^{6}$, Siingh $D .^{3}$ \\ ${ }^{1}$ Department of Physics, Sanjay Ghodawat University Kolhapur, India \\ ${ }^{2}$ Sanjay Ghodawat Group of Institutions, Atigre (Shivaji University, Kolhapur), India \\ ${ }^{3}$ Indian Institute of Tropical Meteorology, Pune, India \\ ${ }^{4}$ Department of BSH, Fabtech Technical Campus, College of Engineering and Research, \\ Sangola, India \\ ${ }^{5}$ Department of Physics, Ahmednagar College, Ahmednagar, India \\ ${ }^{6}$ Regional Remote Sensing Centre-Central, National Remote Sensing Centre, Nagpur, \\ India
}

Total column ozone (TCO) distribution and its variation over the Indian region at different stations for the period of about 30 years from 1986 to 2015 are studied. TCO data is taken from the merged ozone data set (MOD) overpass data for 15 different stations over India. The average correlation between TCO and solar proxies, sunspot number and $\mathrm{F} 10.7 \mathrm{~cm}$ solar flux is more than 0.5 . We further divided the time series of TCO according to solar cycle as 22nd solar cycle (September 1986 to July 996), 23rd solar cycle (August 1996 to November 2008) and 24th solar cycle (December 2008 to December 2015) (on going cycle) for a period of 1986 to 2015. Herein, for long term trend analysis of TCO, we have removed seasonal effect by the deseasonalization process, effects of solar activities, stratospheric waves (quasibiennial-oscillation-QBO and EI Niño-Southern Oscillation-ENSO) by the multifunction linear regression method (MLR). It is found that the direction of the trend in 22nd and the 23th solar cycle is similar while, it is opposite in the 24th solar cycle. We observed a more negative trend in the 22nd solar cycle and less negative trend in the 23rd solar cycle while the trend is positive in the 24th solar cycle. It is found that the role of natural variability is more than that of the ozone depleting substances (ODS) on long term variability in TCO over India. This work presents the variations in trend in TCO with respect to recent three solar cycles.

\section{Century-scale reconstruction of solar activity and a prediction for sunspot cycle 25}

\author{
Nandi D., Bhowmik Pr. \\ Center of Excellence in Space Sciences, India \\ Indian Institute of Science Education and Research Kolkata, India
}

The Sun's activity through its long- and short-term variations impact near-Earth conditions ranging in timescales relevant for space climate to space weather. It is therefore essential to develop predictive models of solar activity, which is governed by the output of the solar dynamo mechanism. However, this has remained a challenging task. Successfully addressing this goal necessitates an understanding of the underlying physics of solar cycle predictability. Building upon the understanding gleaned in the last decade, we have made a prediction for sunspot cycle 25 . In this talk we shall present the understanding that led to this prediction. In particular, we find that the previous cycle poloidal field (which can be quantified as the observed polar flux) is the best predictor of sunspot cycles and that predictions beyond one cycle cannot be achieved with current understanding. Our prediction indicates a weak 
solar cycle 25 similar to the previous cycle; we rule out the possibility of an imminent slide to Maunder minima like conditions over the next cycle.

\title{
A statistical study of co-occurrence of spread $F$ and sporadic $E$ in the middle latitudes
}

\author{
Narayanan V.L. ${ }^{1}$, Balan N. ${ }^{2}$ \\ ${ }^{1}$ National Atmospheric Research Laboratory, India \\ 2 Institute of Space Sciences, Shandong University, China
}

In this work we discuss the statistics of spread $\mathrm{F}$ occurrence in the middle latitude sites in Japan and study the co-occurrence of sporadic $E$ when spread $F$ occurs. We use ionosonde data from four stations namely Okinawa (26.7N geographic; $20.0 \mathrm{~N}$ quasi-dip), Yamagawa (31.2N geographic; $24.5 \mathrm{~N}$ quasi-dip), Kokubunji (35.7N geographic; $28.7 \mathrm{~N}$ quasi-dip) and Wakkanai (45.2N geographic; $38.5 \mathrm{~N}$ quasi-dip) during the year 2014 which is maximum period of the mini solar cycle 24 . The results show that spread $\mathrm{F}$ occurrence peaks during northern summer months in all the four stations. The occurrence of spread $F$ is lesser over Wakkanai compared to the other three stations. Therefore, the maximum spread $F$ occurrence occurs around Kokubunji latitudes above which there is a clear reduction in occurrence of spread $\mathrm{F}$. Moreover, spread $\mathrm{F}$ is often accompanied by sporadic $\mathrm{E}$ layers strengthening theories supporting $\mathrm{E}$ and $\mathrm{F}$ region coupling in generation of spread $\mathrm{F}$ in the middle latitudes. Interesting exception for co-occurrence of sporadic $E$ layers occur during the equinoctial months over Okinawa where more than $80 \%$ of spread $\mathrm{F}$ occurred without accompanying sporadic E. Another exception is spread F over Wakkanai during nonsummer months where $40-60 \%$ of the occurrences are without accompanying sporadic $\mathrm{E}$ layers. These results are discussed in detail on the light of our current understanding on the theories of generation of spread $F$ in low and middle latitudes. The results clearly indicate the gap in our knowledge of middle latitude spread $F$ phenomenon.

\section{Effect of very low frequency (VLF) waves on the acceleration of electrons to relativistic energies}

\author{
Nasi A. ${ }^{1}$, Daglis I.A. ${ }^{1,2}$, Katsavrias Ch. ${ }^{1,2}$, Wen L. ${ }^{3}$ \\ ${ }^{1}$ Department of Physics, National and Kapodistrian University of Athens, Athens, \\ Greece \\ 2 Institute for Accelerating Systems and Applications, Athens, Greece \\ ${ }^{3}$ Center for Space Physics, Boston University, Boston, USA
}

Local acceleration driven by whistler mode chorus waves is fundamentally important for the acceleration of seed electrons in the outer radiation belt to highly relativistic energies. Yet, this mechanism strongly depends on substorm activity and on the source population injected by the substorms into the inner magnetosphere. In this work we use Van Allen Probes data to investigate the relationship between source electrons, seed electrons and chorus waves during periods of increased substorm activity. To that end we calculate the electron phase space density (PSD) for five values of the first adiabatic invariant corresponding to source and seed electrons, during 28 events, out of which, 20 result in enhancement and 8 in 
depletion of relativistic electron fluxes. Furthermore, we perform a superposed epoch analysis in order to compare the time evolution of chorus activity with PSD.

\title{
Participation of BEO Moussala into SEVAN global network
}

\author{
Nikolova N., Arsov T., Angelov I., Angelov Chr. \\ Institute for Nuclear Research and Nuclear Energy, Bulgarian Academy of Sciences, \\ Bulgaria
}

Since 2008 Basic Environmental Observatory (BEO) Moussala is part of SEVAN global network. Its location on highest peak of Balkan Peninsula is make it an appropriate station on which could be measuring the intensity variation of the secondary cosmic rays. Particles with three counting channels - low energy (elecrtron-photon component), neutral particles (mainly neutrons) and high energy particles (HE, mainly muons) are measured by mounted on the top SEVAN detector. It has been developed in the Yerevan Physics Institute (YerPhi) now A. Alikhanian National Laboratory, Armenia. For these more than ten years of operation were detected a several Sun to Earth events.

\section{Practice of research data management in solar-terrestrial physics}

Nosé $M$.

Institute for Space-Earth Environmental Research, Nagoya University

Data-DOI (digital object identifier), data publication, and data citation will promote "Open Science". Recognizing their importance, solar-terrestrial physics (STP) data centers in Japan have been working to mint DOI to their database. We participated from October 2014 in a 1-year pilot program for DOI-minting to science data launched by Japan Link Center, which is one of the DOI registration agencies. In the pilot program, a procedure of the DOI-minting for STP data was established. As a result of close collaboration with Japan Link Center, the first case of data-DOI in Japan (doi:10.17591/55838dbd6c0ad) was created in June 2015. As of April 2019, there are 18 data-DOIs for the STP data in Japan. Four of them are related to geomagnetic field data: the Dst index (doi:10.17593/14515-74000), the AE index (doi:10.17593/15031-54800), the Wp index (doi:10.17593/13437-46800), and magnetotelluric data at Muroto, Japan (doi:10.17593/13882-05900). In the International Association of Geomagnetism and Aeronomy (IAGA), scientists who are working for data centers or observatories started discussion about DOI-minting to their data and a task force was formed in August 2013. In the latest IAGA assembly that was held at Cape Town in August 2017, the task force reported about "Present Status of DOI-minting to Geomagnetic Data/Indices" that is available from https://www.ngdc.noaa.gov/IAGA/vdat/. The report found that there are different types of activities of DOI-minting in individual data centers or observatories. In the field of geophysics including geomagnetism, interests to the DOI-minting are rapidly growing. At the International Union Geodesy and Geophysics General Assemblies that will be held in Montreal, Canada, in July 2019, a joint session entitled "Geoscience data licensing, producing, publication and citation" is planned. This session solicits contributions presenting actual practices and future plans of data licensing, producing, publication, and citation of scientific data, and possible related topics. The international effort will be continued for DOI-minting to scientific data in geophysics. 


\title{
Young Sun, galactic processes and origin life
}

\author{
Obridko V., Ragulskaya M., Khramova E. \\ IZMIRAN, Moscow, Russia
}

The physical conditions in the early Solar system and on Earth, determining the origin, selection and development of the first living systems are discussed. The role of the young Sun dynamics, cosmic rays, galactic processes, geomagnetic fields and other protective shells of the Earth in the formation of the biosphere is emphasized. The selection of a single genetic code, ancient methods of long-term storage of energy and adaptive technologies of the first living systems occurred under the influence of solar, cosmological and geophysical factors. A hypothesis was proposed that the accumulation of energy in polyphosphates could have ensured the survival of the primary biosphere in the conditions of the low luminosity of the young Sun. The hypothesis of a significant influence of oceanic biogenic films and solar radiation on the climate of the early Earth, as well as in the formation of photosynthesis processes, is considered.

\section{Automatic detection and tracking of solar features from the AIA/SDO images - a web tool}

\author{
Pires R. ${ }^{1}$, Fonseca J.M. ${ }^{1}$, Mora A. ${ }^{1}$, Ribeiro R. ${ }^{1}$, Dorotovič $I .{ }^{2,1}$, Rybák J. ${ }^{3}$ \\ ${ }^{1}$ Center of Technologies and Systems - Uninova, Caparica, Portugal \\ 2 Slovak Central Observatory, Hurbanovo, Slovak Republic \\ ${ }^{3}$ Astronomical Institute of the SAS, Tatranská Lomnica, Slovak Republic
}

Solar physics community has benefiting since 2010 from high-resolution and high-cadence solar images provided by the AIA instrument, on-board the SDO satellite. To extract scientific knowledge from huge databases of those images, we need efficient web tools to detect and/or track the solar features. Recently, several research teams have been developing automatic tools to obtain more precise estimations of the solar rotation profile. The objective of this work is to design an automatic and near real-time web tool that detects and tracks coronal bright points (CBPs) on the SDO/AIA FITS images from $19.3 \mathrm{~nm}$ channel, which includes search and visualization functionalities to support astrophysicists performing solar analysis. We combine a Python tool with the Gradient Path Labeling (GPL) segmentation method to identify and track the CBPs to allow calculation of the solar rotation profile, which will be available online in real time. The SDO/AIA images are obtained by querying the Virtual Solar Observatory (VSO) web service.

The preliminary web tool is accessible from the Space-Planetary Interactions Monitoring and Forecasting Laboratory website (SPINLab) in Coimbra (Portugal), under the Solar Data » UNINOVA section, http://www.mat.uc.pt/ obsv/SPINLab. The tool was tested with 403 images, corresponding to 4 days in August 2018. The validation proved that CBPs can be a reliable solar tracking feature and that the web tool includes good detection and tracking capabilities. Hopefully this work will evolve into a more general web tool for investigating the evolution of other solar events and space weather effects. 


\title{
Mechanism of solar flare based on energy storage in the magnetic field of the current sheet
}

\author{
Podgorny I.M. ${ }^{1}$, Podgorny A.I. ${ }^{2}$ \\ ${ }^{1}$ Institute of Astronomy of the Russian Academy of Sciences, Russia \\ 2 Lebedev Physical Institute of the Russian Academy of Sciences, Russia
}

\begin{abstract}
Understanding the solar flare mechanism, which makes it possible to establish a flare situation in the solar corona when using numerical MHD simulations and find a place for the accumulation of magnetic energy for a solar flare, can improve the forecast quality of solar flares, which have a significant impact on the Earth's space environment. The appearance of a flare in the corona at altitudes of 15000 to 30 $000 \mathrm{~km}$ is explained by the accumulation of energy in the magnetic field of the current sheet, which is created in the vicinity of X-type singular. Based on the mechanism of energy release in the current sheet, using the results of numerical simulation and observations, the electrodynamic model of a solar flare is proposed, explaining its main observable manifestations. The hard X-ray emission is caused by the electrons bremsstrahlung in the chromosphere, which are accelerated in fieldaligned currents generated by the Hall electric field. To study the flare situation, numerical MHD simulation was performed in corona above the real active region. At setting the conditions of simulation, no assumptions were done about the flare mechanism. The simulation shows the appearance of a current sheet whose position coincides with the position of the observed source of thermal X-ray emission. For more accurate simulation in real scale of time, the parallelizing of calculations is currently underway on a supercomputer specially assembled for solving this task based on the Tesla M2050 multiprocessor GPU graphics card.
\end{abstract}

\section{Long-term changes in the intensity of mesoscale variations in hydroxyl rotational temperature near the mesopause as indicators of dynamic processes in the underlying atmosphere}

\author{
Popov A.A. ${ }^{1}$, Gavrilov N.M. ${ }^{1}$, Perminov V.I. ${ }^{2}$, Pertsev N.N. ${ }^{2}$, Medvedeva I.V. ${ }^{3}$ \\ ${ }^{1}$ Saint-Petersburg State University, Atmospheric Physics Department, St. Petersburg, \\ Russia \\ 2 Institute of Atmospheric Physics, Russian Academy of Science (IAP RAS), Moscow, \\ Russia \\ ${ }^{3}$ Institute of Solar-Terrestrial Physics, Siberian Branch of Russian Academy of Sciences \\ (ISTP SB RAS), Irkutsk, Russia
}

We used digital difference filters for the analysis of the spectral observations of the rotational temperature of vibrationally excited hydroxyl at altitudes $85-90 \mathrm{~km}$ at the Zvenigorod scientific station of IAP RAS ( $\left.56^{\circ} \mathrm{N}, 37^{\circ} \mathrm{E}\right)$ in 2004-2016 and at the Geophysical observatory of ISTP SB RAS (52 ${ }^{\circ} \mathrm{N}, 103^{\circ} \mathrm{E}$, Tory) in 2012-2017. Seasonal and interannual changes in the mean temperature and intensity of variations with periods $0.4-5.4 \mathrm{~h}$ are studied. These changes may be associated with the propagation of internal gravity waves (IGWs) in the mesopause region. To filter out mesoscale variations, differences between the sequential values of the $\mathrm{OH}$ temperature, averaged over the intervals by duration from $10 \mathrm{~min}$ to $2 \mathrm{~h}$, were obtained. It was revealed, that the average monthly variances of mesoscale variations in the $\mathrm{OH}$ rotational temperature obtained at the Tory station are larger than in Zvenigorod. Average seasonal changes of relative mesoscale variances 
demonstrate two maxima in winter and in summer, with the summer maximum shifted closer to spring months for the Tory station. The reasons for the differences could be different orography and jet streams in the lower and middle atmosphere, also different spectra of IGW horizontal wavelengths due to different geometry of observations at the Tory and Zvenigorod stations.

This study was supported by the Russian Basic Research Foundation \#17-0500458.

Experimental data for Tory station were recorded by the equipment of the 'Angara' Center for Common Use (http://ckp-rf.ru/ckp/3056/) at the ISTP SB RAS within the Basic Research program II.16.

\title{
Solar flare effects on the thermosphere and ionosphere
}

\author{
Qian L. ${ }^{1}$, Woods T.N. ${ }^{2}$ \\ ${ }^{1}$ High Altitude Observatory, National Center for Atmospheric Research, USA \\ ${ }^{2}$ Laboratory for Atmospheric and Space Physics, University of Colorado Boulder, USA
}

Solar flares are sudden increased brightness from the Sun's active regions. This sudden brightness is mainly in X-ray and extreme ultra-violet wavelengths. Solar flares typically last for less than an hour, and take several minutes to arrive at the Earth. During solar flares, solar irradiance increases rapidly in X-rays and extreme ultra-violet. Solar irradiance in these short wavelengths ionizes the thermosphere and creates ionosphere. Therefore, solar flares impact the thermosphere and ionosphere. In this paper, we use model simulations and data to illustrate and understand solar flare effects in the thermosphere and ionosphere system; how flare characteristics affect flare responses in the thermosphere and ionosphere; the EUV late phases of solar flares and how the EUV late phases impact the thermosphere and ionosphere; and large-scale traveling atmospheric disturbances during concurrent solar flare and geomagnetic storm events.

\section{Preliminary observational investigation of equatorial plasma bubbles over Africa using an all-sky airglow imager and the study of its occurrence frequency}

\author{
Rabiu B. ${ }^{1}$, Okoh D. ${ }^{1}$, Shiokawa K. ${ }^{2}$, Otsuka ${ }^{2}{ }^{2}$, Segun B. ${ }^{3}$, Falayi E. ${ }^{4}$ \\ ${ }^{1}$ Center for Atmospheric Research, National Space Research and Development Agency, \\ Abuja, Nigeria \\ 2 Institute for Space- Earth Environmental Research, Nagoya University, Nagoya, Japan \\ ${ }^{3}$ Department of Physics, University of Lagos, Lagos, Nigeria \\ ${ }^{4}$ Department of Physics, Tai Solarin University of Education, Ijebu Ode, Nigeria
}

The first all-sky imager in Africa was installed in Abuja, Nigeria (Geographic: $8.99^{\circ} \mathrm{N}, 7.38^{\circ} \mathrm{E}$; Geomagnetic: $1.60^{\circ} \mathrm{S}$ ), close to magnetic equator, in June 2015 during VarSITI campaign. The camera, has a $180^{\circ}$ fish eye view covering almost the entire airspace of Nigeria. This report covered June 2015 to 31 December 2018. We investigated the occurrence frequency of equatorial plasma bubbles and their dependences on local time, season, and geomagnetic activity. Rate of Change of Total Electron Content ROTI obtained from GNSS observation were also engaged as proxy of plasma bubbles within the coverage of the all-sky imager. Plasma bubble 
occurrence replicated a seasonal variation with semiannual maxima. Most plasma bubble occurrences are found during equinoxes and least occurrences during solstices. The plasma bubbles become more frequent around local midnight and less frequent as we move away from midnight. Majority of the plasma bubbles were observed under relatively quiet geomagnetic conditions (Dst $\geq-40$ and $\mathrm{Kp} \leq 3$ ), but there was no significant pattern observed in the occurrence rate of plasma bubbles as a function of geomagnetic activity.

\title{
Present and future opportunities for geospace science research in the USA
}

\author{
Roussev I.I. \\ National Science Foundation, USA
}

For over two decades, the Geospace Section within the Division of Atmospheric and Geospace Sciences of the USA's National Science Foundation has been providing outstanding opportunities for capacity building for the solar and space sciences communities of the Nation, such as the first National Space Weather Strategy (1995), Faculty Development in Space Sciences (2004), and CubeSats (2007), to name a few. In 2016, the Geospace Section completed a comprehensive Portfolio Review (ICCGS: Investments in Critical Capabilities for Geospace Science 2016 to 2025), which provides strategic guidance for present and future funding initiatives to be undertaken by the Section during 2016-2025. This includes the new Integrative Geospace Science Program and Grand Challenge Projects Program, which are to commence as early as 2020 . This talk provides an overview of the present and future funding opportunities within the Section that support emerging areas of fundamental research in Geospace Science.

\section{Solar energetic electrons and radio emission signatures}

\author{
Samwel S.W.' Miteva R. ${ }^{2}$ \\ ${ }^{1}$ National Research Institute of Astronomy and Geophysics (NRIAG), 11421 Cairo, \\ Egypt \\ ${ }^{2}$ Space Research and Technology Institute - Bulgarian Academy of Sciences, Bulgaria \\ We present two comprehensive catalogs of in situ observed energetic electrons \\ and their related radio emissions. The solar energetic electron events are detected \\ by ACE/EPAM instrument over the period 1997-2017 with energy coverage 103-175 \\ $\mathrm{keV}$. The onset time, peak time and peak intensity are evaluated, in addition to the \\ associated solar flares and coronal mass ejections (CMEs). The related radio emission \\ signature catalog is based on data observed remotely (both from satellites and \\ ground-based observatories) using available quick-look radio plots and observatory \\ reports, as well as single-frequency records provided by the RSTN networks and \\ imaging information from Nancay and Nobeyama Radio Heliographs. We describe \\ the procedures utilized for both catalogs for identifying the electron events, the \\ association to their solar origin and the corresponding radio signatures (radio burst \\ types, single frequency records, dynamic radio spectra). The statistical relationships \\ between the energetic electron events and their solar origin, in addition to the \\ corresponding radio signatures are reported. Thus, for the first time the radio
}


signatures of electrons can be directly compared with the in situ observed electron events over nearly two solar cycles.

\title{
Possible non-linear interactions between some space weather parameters
}

\author{
Sarp V., Kilcik A.
}

Akdeniz University, Faculty of Science, Department of Space Science and Technologies, 07058, Antalya, Turkey

Identifying dynamic interactions is important for modeling of complex systems. Variables can be positively coupled at some times but at other times they appear unrelated or even negatively coupled depending on states of the complex system. So, causation should be distinguished from correlation to determine the interacting variables of the complex system. Here we used Convergent Cross Mapping (CCM) to examine the dynamic relationship among Solar Wind Speed (SWS), Disturbance Storm-Time Index (DST), Solar radio flux at $10.7 \mathrm{~cm}$ (F10.7), Cosmic Ray intensities (CRI) and the Maximum CME Speed Index (MCMESI) for the last two solar cycles (1996-2018). Our results demonstrate the strong coupling between F10.7 and CRI. Their CCM scores also suggest the top-down control by the F10.7 is much stronger than the bottom-up control by CRI. This asymmetrical bidirectional coupling is consistent with current cosmic ray transport models. A symmetrical bidirectional coupling with equivalent strength is also found between F10.7 and the MCMESI. On the other hand, the result of unidirectional weak coupling between F10.7 and DST suggests F10.7 can affect the dynamics of DST, but DST has no effect on F10.7. Lastly, SWS and DST found to be interacted with a moderate asymmetrical bidirectional coupling which suggest the effects of SWS is much more detectable on DST dynamics than vice versa.

\section{Prediction of solar wind parameters at 1 AU using an artificial neural network and multiple observations}

\author{
Shen F., Yang Y., Feng $X$. \\ SIGMA Weather Group, State Key Laboratory of Space Weather, National Space \\ Science Center, Chinese Academy of Sciences, Beijing, China
}

In this research, we present a hybrid artificial neural network (ANN) model, which applied an ANN with observational and theoretical input, to predict solar wind parameters at $1 \mathrm{AU}$. The model is a hybrid system merging various observational and theoretical information as input. Different inputs are tested including individual parameters and their combinations in order to select an optimum. Then, the optimal model is implemented for prediction. The modeling results are validated by both error analysis and event-based analysis from 2007 to 2016. Our results demonstrate that the HISS model can predict the large-scale solar wind speed at $1 \mathrm{AU}$ well for year 2007 to 2016 . The HSE analysis shows our model can predict $68.2 \%$ of the observed HSEs. The artificial intelligent techniques have been greatly improved in the last few years, with the explosion of deep learning. It can be expected that the solar wind prediction will be greatly improved with more advanced machine learning techniques and more observational data collected. 


\title{
Recent Results of the ground-based multi-point network observation of the upper atmosphere, ionosphere, and magnetosphere by the OMTIs and the PWING project
}

\author{
Shiokawa K.1, Otsuka Y.1, and PWING Team ${ }^{2}$ \\ ${ }^{1}$ Institute for Space-Earth Environmental Research (ISEE), Nagoya University, Japan \\ 2 PWING Team: http://www.isee.nagoya-u.ac.jp/dimr/PWING/en/
}

The Institute for Space-Earth Environmental Research (ISEE), Nagoya University, Japan operates ground-based multi-point network for measurements of the upper atmosphere, ionosphere, and magnetosphere, mainly through the OMTIs and the PWING project. The Optical Mesosphere Thermosphere Imagers (OMTIs) consist of about twenty all-sky cooled-CCD airglow imagers, five Fabry-Perot interferometers, three airglow temperature photometers, and three meridian-scanning photometers. They measure two-dimensional airglow images in the mesopause region and in the thermosphere, wind and temperatures in the lower thermosphere, and airglow rotational temperatures in the mesopause region. Details of the OMTIs project can be seen at http://stdb2.isee.nagoya-u.ac.jp/omti/. The PWING stands for 'study of dynamical variation of Particles and Waves in the INner magnetosphere using Ground-based network observations. ' The PWING project started on April 2016 as a 5-year project of the Grant-in-Aid for Specially Promoted Research of the Japan Society for the Promotion of Science (JSPS) (16H06286). The PWING project deploy OMTIs all-sky cooled-CCD airglow imagers, $64-\mathrm{Hz}$ sampled induction magnetometers, $40-\mathrm{kHz}$ VLF receivers, and $64-\mathrm{Hz}$ riometers at 8 stations at magnetic latitudes of $\sim 60$ degree around the north-pole to cover longitudinal variation of aurora and electromagnetic disturbances in the inner magnetosphere. Details of the PWING project can be seen at http://www.isee.nagoyau.ac.jp/dimr/PWING/PWING_web_e.htm. These PWING and OMTIs instruments are in automatic operation at various locations from high to equatorial latitudes in Canada, US (Alaska), Russia, Norway, Finland, Iceland, Japan, Thailand, Indonesia, Nigeria, and Australia. In the presentation, we introduce current status and some recent results obtained by these multi-instrument ground networks around the world.

\section{Predictability of a variable source of high speed streams of ultrarelativistic particles based on the simulation analysis}

\author{
Shkevov R. ${ }^{1}$, Erokhin N.S. ${ }^{2}$, Loznikov V.M. ${ }^{2}$, Zolnikova N.N. ${ }^{2}$, Mikhailovskaya L.A. ${ }^{2}$ \\ ${ }^{1}$ Space Research and Technology Institute - Bulgarian Academy of Sciences, Bulgaria \\ ${ }^{2}$ Space Research Institute - Russian Academy of Sciences, Russia
}

Origin and predictability of the high speed streams of ultrarelativistic particles are among the most important and basic tasks of the Earth's space environment exploration. We are tempted to prove that an effect not studied well till now, as charged particles surfatron acceleration is one of the possible mechanisms for generating high speed streams of ultrarelativistic particles. A theoretical investigation of this acceleration mechanism by numerical experiments is presented in order to understand and support the hypothesis. Simulation analysis of the resonance acceleration of electrons and protons in the heliosphere periphery is presented. The study is done by an exact solution of second order nonlinear nonstationary equations for the wave phase on the particle's trajectory. Detailed 
examination of the particles acceleration parameters and its elements is presented in graphical form. Theoretical results are discussed, taking into account the information in existing databases from satellite experiments. Conclusions about predictability of a variable source of high speed streams of ultrarelativistic particles with surfatron mechanism origin are made.

\title{
Neutral and plasma density perturbations in the top-bottom-side ionosphere associated with MSTIDs
}

\author{
Suzuki S. ${ }^{1}$, Park J. ${ }^{2}$, Otsuka Y. ${ }^{3}$, Shiokawa K. ${ }^{3}$, Liu H. ${ }^{4}$, Lühr H. ${ }^{5}$ \\ ${ }^{1}$ Faculty of Regional Policy, Aichi University, Japan \\ 2 Korea Astronomy and Space Science Institute, South Korea \\ ${ }^{3}$ Institute for Space-Earth Environmental Research, Nagoya University, Japan \\ ${ }^{4}$ Earth and Planetary Science Division, Kyushu University SERC, Kyushu University, \\ Japan \\ ${ }^{5}$ Helmholtz Centre Potsdam, GFZ, German Research Centre for Geosciences, Germany \\ Medium-scale traveling ionospheric disturbances (MSTIDs) are a well-known \\ wavy structure in the F-region ionosphere. They typically have a horizontal \\ wavelength of several hundred kilometers and a periodicity of about one hour. \\ Although, the MSTIDs were considered to be caused by atmospheric gravity waves, \\ recent studies have suggested that the generation of the MSTID in nighttime is highly \\ associated with coupling processes between the E- and F-region electrodynamics. To \\ confirm the different processes in the MSTID generation in daytime and nighttime, \\ CHAMP satellite measurements would be greatly helpful; CHAMP plasma and neutral \\ density data obtained in the day- and night-side sector can monitor the phase \\ relations between the neutral and ionospheric plasma perturbations simultaneously \\ at the top-side F-region. \\ As the first step, we compared the MSTID signatures between the CHAMP and \\ ground-based 630-nm airglow measurements to validate the MSTID detection by \\ CHAMP. Airglow imaging is a quite useful technique to investigate two-dimensional \\ characteristics of the nighttime MSTIDs. Previous study by Park et al. [2009, JGR] \\ made an investigation of spatial signatures regarding one MSTID event using airglow \\ images along the CHAMP orbit. The ISEE, Nagoya University, has operated airglow \\ imaging network, as the OMTI system; this network gives much more chance to \\ make coordinated measurements with CHAMP. \\ In this presentation, we will report phase relations between the neutral and \\ plasma density perturbations associated with the clear MSTID events in which \\ CHAMP passed over the field-of-views of the OMTI camera.
}




\title{
Equatorial plasma bubble seeding under propagation of MSTIDs and MLT-gravity waves
}

\author{
Takahashi H. ${ }^{1}$, Wrasse C.M. ${ }^{1}$, Figueiredo C.A.O.B. ${ }^{1}$, Barros D. ${ }^{1}$, Abdu M.A. ${ }^{1}$, \\ Otsuka Y.2, Shiokawa K.2, Paulino $I^{2}{ }^{3}$ \\ ${ }^{1}$ Instituto Nacional de Pesquisas Espaciais (INPE), Brazil \\ 2 Institute for Space-Earth Environmental Research (ISEE), Nagoya University, Japan \\ ${ }^{3}$ Universidade Federal de Campina Grande (UFCG), Brazil
}

We observed signatures of dynamical processes from troposphere to ionosphere, which might be responsible for seeding of equatorial plasma bubbles (EPBs). Simultaneous observation of Mesosphere to Lower Thermosphere Gravity Waves (MLT-GWs) by $\mathrm{OH}$ airglow imager, propagating Ionospheric Disturbances (MSTIDs) and Equatorial Plasma Bubbles (EPBs) by groundbased GNSS receivers, OI $630 \mathrm{~nm}$ imager and ionosonde have been carried out in the equatorial and low latitude region of Brazil. On 16-17 September 2015, MSTIDs were observed in the 5 to 25S region in the afternoon to evening period. EPBs started to appear after when the MSTID crossed over the solar terminator at 22:30 UT. Large scale MLT-GWs were also observed at $7 \mathrm{~S}$ and 23S. Besides, a large tropospheric convection system was observed at around 30S. Gravity wave ray-tracing of the observed MLT-GWs indicated that the waves were from the troposphere and could propagate upward in the $\mathrm{F}$ layer bottom height. These observational and model simulations indicate that the gravity waves in the MLT region are coming from the troposphere and responsible for generation of MSTIDs in the ionosphere, and therein, generating a perturbation in the $\mathrm{F}$ layer bottom height resulting EPBs.

\section{CME density derivation from remote sensing stereoscopic data}

\author{
Temmer M. ${ }^{1}$, Holzknecht L. ${ }^{1}$, Dumbovic M.1,2, Vrsnak B. ${ }^{2}$ \\ ${ }^{1}$ Institute of Physics, University of Graz, Austria \\ ${ }^{2}$ Hvar Observatory, Faculty of Geodesy, University of Zagreb, Croatia
}

Coronal mass ejections (CMEs) propagating in the heliosphere are exposed to a drag force due to the ambient solar wind. Mass pile-up in interplanetary space can have strong effects on the drag force, and with that on the CME propagation time and energy input to the magnetosphere. For a sample of well observed events, we determine the de-projected 3D mass and its evolution up to a distance range of about 15Rs using combined STEREO-SECCHI COR1 and COR2 data, for which no pile-up at the CME front is found (see also Bein et al., 2013). Applying the GCS forward fitting model (Thernisien et al., 2006, 2009) on COR2 data, we obtain the volume of the CMEs. Working under the assumption that the CME mass is constant beyond $15 R$ s and that the CME undergoes self-similar expansion, we estimate the CME density at the distance of $1 \mathrm{AU}$. The results are compared to in-situ proton density data measured for the associated ICME's sheath and magnetic structure for which we derive a trend towards a mass increase at the CME front. 


\title{
Atmospheric response to solar proton events in the latest reanalysis data
}

\author{
Tomikawa $Y$.
}

National Institute of Polar Research and SOKENDAI (The Graduate University for Advanced Studies), Japan

Solar proton events (SPEs) have some impacts on the middle atmosphere through NOx/HOx formation, its subsequent ozone destruction, and Joule heating. Several observational and simulation studies have reported their impact on temperature and circulation in the middle atmosphere. However, their impact has never been captured in the meteorological reanalysis data. The latest meteorological analyses have been developed to assimilate satellite radiance and ozone observations in the upper stratosphere and lower mesosphere, so that they have a potential to reproduce atmospheric impacts of SPEs. This study examines whether the SPEs' impacts on the middle atmosphere can be captured in the latest reanalysis data or not.

\section{Sun-to-Earth MHD simulations of large solar eruptions}

Török $T$.

\author{
Predictive Science Inc., San Diego, CA, USA
}

Coronal mass ejections (CMEs) are the main driver of space-weather disturbances in the terrestrial magnetosphere. Predicting the impact of CMEs before they arrive at Earth is one of the main challenges of solar and heliospheric physics. A candidate tool for this purpose are numerical simulations. State-of-the-art MHD simulations are now capable of modeling CMEs all the way from Sun to Earth, but they are computationally still too demanding to be used for real-time modeling. At present, only a simplified model (WAS-ENLIL), which does not include the corona and simulates CMEs as velocity perturbations, is used for operational space-weather forecast. However, given the continuous increase of computing power, more sophisticated models may become available for this purpose in the near future, and first attempts are currently made to prepare such models for operational use. A specific task at hand is to evaluate the accuracy of these simulations in reproducing in-situ measurements at Earth. In this presentation, I will review state-of-the-art CME simulations and discuss their predictive capabilities and limitations. As an example, I will present a recent Sun-to-Earth simulation of the well-known 14 July 2000 'Bastille-Day' event, which produced a very strong geomagnetic storm.

\section{Quantifying ionospheric disturbances for user oriented applications}

\author{
Tsagouri I., Belehaki A., Koutroumbas K., Xenaki I. \\ National Observatory of Athens, IAASARS, Greece
}

The reliable identification of space weather effects on the Earth's ionosphere and the effective communication of ionospheric alerts and warnings in support of technological systems' operations are always reported among key users' 
requirements. These are definitely not straightforward tasks, since the requirements differ for different applications, but also for effects of different temporal and spatial scales (i.e. large-scale and small-scale ionospheric effects) that characterize the complex ionospheric response to solar wind forcing. This paper aims to address relevant needs for short-term ionospheric warning purposes within the European TechTIDE warning system (http://www.tech-tide.eu/). The work presented includes: i) Distinction between large scale perturbations in ionospheric plasma properties (e.g. ionospheric storm effects) and travelling ionospheric disturbances (TIDs) in real time mode. This challenge is addressed through the sophisticated representation of ionospheric background conditions by running averages with variable time window. This window ranges from a few minutes to about 30-days to capture ionospheric disturbances of different scales. ii) Quantification of the ionospheric activity level with respect to the systems' malfunctions. The deviations of the observed values from the background conditions are statistically analyzed to provide the quantitative criteria for the determination of ionospheric activity levels. This task is also supported by the comparison with TID activity indicators related to users requirements, as for example the AATR (Along Track TEC Rate) index. The analysis applies to electron densities at various altitudes in the bottomside and topside ionosphere, and the critical frequency foF2.

\title{
Solar prominence observations during 2017 total solar eclipse
}

\author{
Tsvetkov Ts. ${ }^{1}$, Petrov N. ${ }^{1}$, Myshyakov I. ${ }^{2}$, Miteva R. ${ }^{3}$ \\ ${ }^{1}$ Institute of Astronomy and NAO, Bulgarian Academy of Sciences, Bulgaria \\ 2 Institute of Solar-Terrestrial Physics, SB Russian Academy of Sciences, Russia \\ 3 Space Research and Technology Institute, Bulgarian Academy of Sciences, Bulgaria
}

Total solar eclipses provide an opportunity to observe faint coronal structures such as prominences. Our team successfully captured three prominences visible at the base of helmet streamers during the 2017 August 21 total solar eclipse, observed from the territory of the state of Oregon, USA. Images are processed in order to achieve best visualization. Obtained observational material as well as AIA/SDO sets of data with enhanced corona (summing the off-limb component of several images) reveal details about prominence environment. Magnetic field reconstruction is also used to analyze results. They are compared with previous total solar eclipse observations from 1999 and 2006.

\section{D reconstruction of interacting CMEs and related SEP characteristics}

\author{
Tsvetkov Ts. ${ }^{1}$, Temmer M. ${ }^{2}$, Miteva . $^{3}$ \\ ${ }^{1}$ Institute of Astronomy and NAO, Bulgarian Academy of Sciences, Bulgaria \\ ${ }^{2}$ Institute of Physics, University of Graz, Austria \\ ${ }^{3}$ Space Research and Technology Institute, Bulgarian Academy of Sciences, Bulgaria
}

Coronal mass ejection (CME)-driven shocks are among the physical processes that accelerate solar energetic particles (SEPs) in the corona and interplanetary space. Studies on whether the collision of two or more CMEs could influence the SEP characteristics lead to contradictory results in the past. In the present study we address the issue of interacting CMEs using combined data from SOHO LASCO and 
STEREO SECCHI coronagraphs to derive the 3D geometry and kinematics. We relate the obtained CME 3D parameters to the occurrence and magnitude of the SEP events. The contribution of other factors such as associated flares and source locations are also considered.

\title{
Characterization of coronal mass ejections from the associated high frequency type II solar radio bursts
}

\author{
Umuhire A.C. ${ }^{1}$, Uwamahoro J. ${ }^{2}$, Gopalswamy N. ${ }^{3}$, Mungufeni P. ${ }^{4}$, Monstein C. ${ }^{5}$ \\ 1 University of Rwanda, College of Education, Rwanda \\ 2 University of Rwanda, College of Education, Rwanda \\ 3 NASA Goddard Space Flight Center, USA \\ ${ }^{4}$ Mbarara University of Science and Technology, Uganda \\ ${ }^{5}$ Institute for Astronomy-ETH Zurich, Switzerland
}

Solar Radio Bursts (SRBs) are often early indicators of incoming geo-effective space weather events. This study present first results obtained to characterize CMEs using associated High Frequency SRBs observed from the ground using CALLISTO spectrometers. For the analysis, CALLISTO SRBs data in the form of dynamic spectra were used, while the daily report of SRBs were used for events verification. In the period between 2010 and 2017, this study found 47 high frequency SRBs out of 176 (26\%) could be detected by the ground CALLISTO spectrometers network. Using the drift rate of analyzed SRBs events, the CME speed of associated CMEs could be derived with $98 \%$ accuracy. Further, a better correlation (96\%) was found between the CME height and the starting frequency of analyzed type II SRBs. These estimate were confirmed by analyzing the spatio-temporal evolution of one arbitrary event observed by both STEREO and SOHO spacecraft. This study confirms the importance of space weather monitoring from ground using CALLISTO spectrometers.

\section{Prominence eruption triggered by coronal rain in the solar atmosphere observed by SDO/AIA and STEREO/EUVI}

\author{
Vashalomidze Z.1 ${ }^{1}$ Zaqarashvili T.V. ${ }^{1,2,3}$, Kukhianidze V. ${ }^{1}$ \\ ${ }^{1}$ Abastumani Astrophysical Observatory at Ilia State University, Tbilisi, Georgia \\ ${ }^{2}$ Space Research Institute, Austrian Academy of Sciences, Graz, Austria \\ ${ }^{3}$ IGAM-Kanzelhohe Observatory, Institute of Physics, University of Graz, Graz, Austria
}

Triggering process for Coronal Mass Ejection (CME) in the solar atmosphere is not fully understood. We use multi-spacecraft and multi-wavelength observations to detect the process of prominence/filament instability and consequent eruption of CME. We use time series of $304 \AA, 171 \AA, 193 \AA$ and $211 \AA$ spectral lines from SDO spacecraft and $304 \AA, 171 \AA, 195 \AA$ and $284 \AA$ spectral lines from STEREO spacecraft. Prominence/filament system has been observed during November 08-23, 2011, from different angles by SDO, STEREO_A and STEREO_B. Observations show that a giant tornado started to rise up near the prominence leg at 20:00 UT, November 20, which later caused coronal rain blobs (at UT 16:00, November 21) flowing downwards from the prominence main body. The coronal rain continued till UT 20:20, November 22, and triggered the instability of the prominence, which erupted as a CME at UT 22:30, November 22. We suppose that the mass loss due to the coronal rain may lead to the instability of prominence and to the consequent eruption of CME. If confirmed, 
coronal rain observations from prominence main body could be used for space weather predictions.

\title{
Temporal variability of solar activity influences on the lower atmosphere and its possible reasons
}

\author{
Veretenenko S., Ogurtsov $M$.
}

Ioffe Institute, St. Petersburg, Russia

\begin{abstract}
Temporal variability of solar activity influences of the lower atmosphere state remains one of unresolved problems of solar-terrestrial physics. In this work we continue studying this variability and its possible reasons. It was shown that temporal variations of correlation links between troposphere pressure at extratropical latitudes and solar activity characteristics depend of the large-scale circulation regime which, in turn, is closely related to the strength of the stratospheric polar vortex. The correlation reversals were found to coincide with turning points in the evolution of the large-scale circulation forms according to the Vangengeim-Girs classification, these points accompanying transitions between strong and weak states of the vortex at the multi-decadal time scale. It was shown that the changes of the polar vortex intensity may be due to long-term variations in total solar irradiance and geomagnetic activity.
\end{abstract}

\section{Long-term variations in occurrence frequency of magnetic storms with sudden and gradual commencements}

\author{
Veretenenko S. ${ }^{1}$, Ogurtsov M. ${ }^{1}$, Obridko V. ${ }^{2}$ \\ ${ }^{1}$ Ioffe Insitute, St. Petersburg, Russia \\ 2 IZMIRAN, Moscow, Russia
}

The study of long-term variations in frequency of occurrence of magnetic storms with sudden and gradual commencements was carried out, the data from IZMIRAN and Slutsk (Pavlovsk) magnetic observatories for the period 1878-2015 being used. It was found that annual occurrences of large and moderate magnetic storms with gradual commencement are characterized by a pronounced long-term variability, their wavelet spectra revealing dominant periodicities of about 35 years (the Bruckner cycle) on the entire time interval under study. Occurrences of large, moderate and small magnetic storms with sudden commencement reveal strong 11yr harmonics, whereas long-term variations are insignificant. As magnetic storms with sudden and gradual commencements are related to different agents of solar activity (CMEs and high-speed streams from coronal holes), the obtained results provide evidence for a different temporal evolution of local and global solar magnetic fields responsible for these agents. 


\title{
Role Of the Sun and the Middle atmosphere/thermosphere/ionosphere In Climate (ROSMIC): A retrospective and prospective view
}

\author{
Ward W., Lübken F.-J., Seppala A., Funke B., Krivolutsky A., Woods T.N., \\ Nakamura T., Stolle Cl., Yigit E., Lastovicka J. \\ University of New Brunswick, Canada \\ Leibniz Institute of Atmospheric Physics, Germany \\ University of Otago, New Zealand \\ Instituto de Astrofisica de Andalucia, Spain \\ Central Aero-logical Observatory, Russia \\ LASP, USA \\ National Institute of Polar Research, Japan \\ GFZ German Research Centre for Geosciences, Germany \\ George Mason University, USA \\ Institute of Atmospheric Physics, AS CR, Czech Republic \\ NCAR, USA \\ Physical Research Laboratory, India
}

While knowledge of the energy inputs from the Sun (as it is the primary energy source) is important for understanding the solar-terrestrial system, of equal importance is the manner in which the terrestrial part of the system organizes itself in a quasi-equilibrium state to accommodate and re-emit this energy. The ROSMIC project, has been the component of SCOSTEP's Variability of the Sun and Its Terrestrial Impact (VarSITI) program which supported research into the terrestrial component of this system. The four themes supported under ROSMIC are Solar Influence on Climate, Coupling by Dynamics, Trends in the MLT and Trends and Solar Influence in the Thermosphere. Over the course of the VarSITI program, scientific advances were made in all four themes. This included improvements in understanding 1 ) the transport of photochemically produced species from the thermosphere into the lower atmosphere, 2) the manner in which waves produced in the lower atmosphere propagate upward and influence the winds, dynamical variability and transport of constituents in the mesosphere, ionosphere and thermosphere, 3 ) the character of the long term trends in the mesosphere and lower thermosphere and 4) the trends and structural changes taking place in the thermosphere. This paper reviews this progress and summarizes the future research directions anticipated in these areas in the future. The effects changes to the Sun and changes to the terrestrial system (such are currently occurring as a result of anthropogenic influences) may have, need to be understood to support and guide future human activities on the Earth.

\section{Data-led study on the Sun-Earth variability and its relationship to socio-economic activity in $18^{\text {th }}-19^{\text {th }}$ centuries including the Dalton minimum}

\author{
Watanabe $T$.
}

ICS WDS International Programme Office, Koganei 184-8795, Japan

In the Open-Science era, assuring easy multi-disciplinary usage of data is crucial to create new data-led research field. The influence of solar-terrestrial variability on climate and human activities will be a typical subject attracting many scientists in a 
wide variety of disciplines. However, there still exist many obstacles in multidisciplinary data usage. To specify the problems, a trial is performed by selecting the interval of 18th-19th Centuries to access to environmental and socio-economic data. This interval was in the post Maunder Minimum (MM) era including the Dalton Minimum (DM) in 1790-1830. Since the anthropological warming was not important yet in these centuries, this interval will be pertinent to study "basic" characteristics of solar-climate relationship although we should take into account the influence of high volcanic activity in this interval, e.g. Tambola in 1815. In the DM period, the global temperature was generally low, producing reduction of the crop yield. However, the direct connection between the cold climate in DM and the economic situation particularly in Europe is not clear in this stage because of the influence of quick changes in the socio-economic environment (e.g. French Revolution, Napoleonic War, Industrial Revolution), Concerning data, we need to pay effort to produce well documented ready-to-use data sets expecting usages by scientists out of the field. Many important data are only available in printed records or documents, but they are missing now. Preservation of these data in machine-readable formats is urgent. Involvement of dedicated citizen scientists will be important in the data rescue.

\title{
Sunspot number prognoses for the $24^{\text {th }}$ and $25^{\text {th }}$ solar cycle
}

\author{
Werner R., Guineva V.
}

Space Research and Technology Institute - Bulgarian Academy of Sciences, Bulgaria

The solar activity changes have important impacts on space weather and on the Earth atmospheric processes. The prognosis of the solar activity is a key issue of the VARSITI project. A substantial contribution was made for the application of wellknown prediction methods, their further development and the development of new methods for the forecast of the solar activity. A lot of solar activity prognoses were done based on the Sunspot number series by different methods. VARSITI members have published early and current predictions for the next solar cycle in scientific journals. Here an overview of the Sunspot number predictions is given. For the 24th solar cycle the predictions are compared with the real Sunspot number observations.

According to our own prognosis based on the old version of the Sunspot number data set a sunspot number maximum of 90 was expected for the 24th solar cycle to occur in 2013. Using the new Sunspot number semi-annual SILSO data it was obtained that the Sunspot number will be in the range from 65 up to 185 with a likelihood of $95 \%$. The maximum of 117 for the 25th solar cycle will be reached in the first half of 2023.

\section{Solar cycle variation of the heliospheric plasma sheet thickness}

\author{
Wu C. $-{ }^{1}{ }^{1}$, Kan L. ${ }^{2}$, Lepping R.P. ${ }^{3}$ \\ ${ }^{1}$ Naval Research Laboratory, Washington, DC, 20375, USA \\ 2 Johns Hopkins Applied Physics Laboratory, Laurel, Maryland, 20723, USA \\ 3 UMBC/NASA/GSFC, Greenbelt, Maryland, USA
}

We conduct a survey of the previous heliospheric plasma sheet (HPS) data sets and find a solar cycle dependence - where the width of the HPS tends to be wider during solar minimum years and narrower during solar maximum years. The HPS is 
thicker near solar minimum than near solar maximum by a factor of 1.6 (in solar cycle 23) - 8 (in solar cycle 24). We also found that the averaged HPS thickness in 2007 (near the minimum of solar cycle 23/24) was almost ten times larger than that in 1995 (near minimum of solar cycle 22/23), and was associated with a weak polar magnetic field in 2007. Based on the solar surface field measurements, we found that the average solar magnetic field strength $(|B|)$ at 2.5 solar radii (Rs) was $\sim 40 \%$ larger in 1995 than in 2007 (0.22 gauss vs 0.16 gauss). We also found a larger ( $\sim 27 \%)$ magnetic pressure gradient force in 1995 than in 2007 . Because this gradient force points toward the equator in a low-beta coronal plasma (which is also probably true outward of the Sun), a wider HPS is expected to occur in 2007 than in 1995, at least close to the Sun. This result supports the so-called heliospheric plasma sheet inflation hypothesis - i.e. the HPS is wider if the Sun's polar field is weaker, and narrower if the Sun's polar field is stronger.

\title{
The 04-10 September 2017 Sun-Earth connection events: Solar flares, coronal mass ejections/magnetic clouds, and geomagnetic storms
}

\author{
Wu C. $-{ }^{1}{ }^{1}$, Kan L. ${ }^{2}$, Lepping R.P. ${ }^{3}$, Lynn H. ${ }^{1}$ \\ ${ }^{1}$ Space Science Division, Naval Research Laboratory, Washington, DC 20375, USA \\ 2 Space Department, Applied Physics Laboratory, Laurel, Maryland, USA \\ 3 UMBC, GSFC/NASA, Greenbelt, Maryland, USA
}

In early September of 2017, a series of solar flares and CMEs erupted from the Sun. The SECCHI-Cor2a coronagraph recorded two Sun-Earth directed CMEs on September 4 (referred to as CME04) and 6 (CME06). The Wind spacecraft ( 212.839 solar radii, Rs) recorded two interplanetary shocks, presumably driven by CME04 and CME06, at 22:41UT on 6-09-2017 (Shock06) and at 22:48 UT on 7-092017 (Shock07), respectively. The traveling time of the CME04/Shock06 ( $\Delta$ tshockCME@18Rs) and CME06/Shock07 from 18 Rs to the Wind spacecraft was 41.52 and 32.47 hours. The propagating speed of the CME04 and CME06 at 18 Rs were 886 and $1368 \mathrm{~km} / \mathrm{s}$, respectively. The estimated $\Delta$ tshock-CME@18Rs is 42.45 and 27.5 hours for CME04 and CME06, respectively. This simple estimate of the CME propagation speed provides a satisfactory result for the CME04 event (error 8\%) but not for the CME06 event (error 15.3\%). The CME06 was delayed due to an interaction with the preceding event. It is suggested that the CME speed estimated near the Sun with coronagraph images can be a good estimator for the ICME transit time when there is no pre-event. A three-dimensional magnetohydrodynamic simulations is performed to address this issue by providing a panoramic view of the entire process not available from the observations. 


\title{
White-light signatures of shocks driven by coronal mass ejections and interplanetary type II radio bursts
}

\author{
Yashiro S.1,2, Gopalswamy N.2, Akiyama S.1,2, Mäkelä P.1,2 \\ ${ }^{1}$ Catholic University of America, USA \\ 2 NASA Goddard Space Flight Center, USA
}

\begin{abstract}
We report on the relationship between shock signatures observed by the Large Angle and Spectrometric Coronagraph (LASCO) on Solar and Heliospheric Observatory (SOHO) and interplanetary (IP) type II radio bursts observed by the WAVES instrument on the Wind spacecraft. Diffuse features surrounding coronal mass ejections (CMEs) in white-light (WL) coronagraph images are visible signatures of shocks. In order to investigate the properties of the WL shock signatures, we examined $95 \mathrm{limb}$ CMEs with a speed of $900-1200 \mathrm{~km} / \mathrm{s}$ recorded in the SOHO LASCO CME Catalog. We found that $39 \%$ (37/95) of $1,000 \mathrm{~km} / \mathrm{s}$ CMEs had the WL shock signature at the front (and the flank), 35\% (33/95) had the one at the flank only, and $26 \%$ (25/95) did not have the WL signatures. The association rate of the IP type II bursts was $41 \%$ (or $15 / 37$ ) for the front shocks but $12 \%$ (or $4 / 33$ ) for the flank shocks. Two IP type II bursts were found in the 25 CMEs lacking the WL shock signatures. The higher correspondence between the WL shocks and IP type II bursts suggests that the shock near the CME nose is preferable site for the electron acceleration.
\end{abstract}

\section{Properties of interplanetary drivers of magnetospheric disturbances}

Yermolaev Yu.I., Lodkina I.G., Yermolaev M.Yu., Borodkova N.L., Riazantseva M.O. Space Research Institute (IKI), Russian Academy of Sciences, Russia

Using our catalog of large-scale solar-wind (SW) phenomena for 1976-2017 (see site ftp://ftp.iki.rssi.ru/pub/omni/ and paper by Yermolaev et al., 2009) and OMNI measurements of plasma and magnetic field parameters, we study the average temporal profiles of interplanetary drivers of magnetospheric disturbances: corotating interaction regions (CIRs), interplanetary coronal mass ejections (ICMEs, including both magnetic clouds (MCs) and ejecta), and sheaths as well as interplanetary shocks (ISs). We show that two types of compression regions are formed by the same physical mechanism, regardless of the type of piston (highspeed stream (HSS) for CIR or fast ICME for sheath). One of the possible reasons behind the observed differences in the parameters in MCs and ejecta is that during ejecta observation, the satellite passes farther from the nose of the area of ICME than when MCs are observed. We also discuss the longitude (relative to ecliptic plane) angle and helium abundance variations in different SW types. Different properties of drivers result in different reaction of magnetosphere. Storm generation process is most effective for sheaths and it is necessary to take into account for study/prediction of impact for complex phenomena including sequence 'shock + sheath + ICME'. 


\title{
Coupling of meteorology severe weather and space weather via the excitations and propagations of gravity waves
}

\author{
Yue J., Xu S., Xue X., Vadas Sh.L., Miller S.D., Azeem I., Straka III W., \\ Hoffmann $L$. \\ Hampton University \\ University of Science and Technology of China \\ NWRA \\ Colorado State University \\ National Science Foundation \\ University of Wisconsin Madison \\ Julich Supercomputing Center
}

In this presentation, I will present how the lower atmosphere, upper atmosphere and the ionosphere are connected via the excitations and propagations of gravity waves. During Hurricane Matthew in September/October 2016, a photographer at Puerto Rico captured photos of sprites and airglow gravity waves at nights. Sprites were linked to lightning strikes and gravity waves were excited by deep convection in the hurricane. Same gravity waves also display in the stratosphere (AIRS observations) and mesosphere (Suomi NPP VIIRS DNB observations). Over the late $r$ phase of Matthew, concentric traveling ionospheric disturbances were seen in GPS TEC maps in Eastern US on Oct 6/7/8. These comprehensive and concurrent observations demonstrate the coupling of the hurricane/ocean with the upper atmosphere of mesosphere and ionosphere. Space weather is linked to the severe weather over the ocean.

\section{Source regions identification and geophysical effects of stealth coronal mass ejections}

\author{
Zagainova Yu.S. ${ }^{1}$, Fainshtein V.G. ${ }^{2}$, Gromova L.I. ${ }^{1}$, Gromov S.V. ${ }^{1}$ \\ ${ }^{1}$ Pushkov Institute of Terrestrial Magnetism, Ionosphere and Radio Wave Propagation, \\ Russian Academy of Sciences, Solar Physics and Solar-Terrestrial Relations, Russia \\ ${ }^{2}$ Institute of Solar-Terrestrial Physics of Siberian Branch of Russian Academy of \\ Sciences, Russia
}

Coronal mass ejections (CMEs) are generally associated with eruptive phenomena or low coronal signatures (LCSs) in the lower corona such as solar flares, filament eruptions, extreme ultraviolet (EUV) waves, magnetic reconfiguration, or jets. The present state the stealth CMEs as events without LCSs may be of interest applied problems related to tracking geoefficiency of these events and prediction of so called problem geomagnetic storms, i.e. storms without a clearly observed solar source. Stealth CMEs observe in the field of view of SOHO and STEREO coronagraphs. But the important problem is identification of source regions and geophysical effects of these type mass ejections. We present new approach to search of traceable source of stealth CMEs on solar disk. We found that the stealth-CMEs were accompanied by various manifestations of short-time small-scale activities in the place of the CME assumed formations basing on image analysis from different EUV channels. We registered motion of small-scale loops (or loop-like structures) and the formation of stealth CMEs frontal structures (FS) as a next step. Magnetic field variations in the regions of stealth CMEs formation were studied also. Time velocity profiles of stealth CMEs FS are determined. We supposed that the stealth CMEs effect on the Earth 
magnetosphere did not lead to a noticeable geomagnetic field disturbances described by the Dst-index, but increase the auroral activity (AL index).

\title{
ISEST/Minmax24 project: International Study of Earth-affecting Solar Transients
}

\author{
Zhang J. ${ }^{1}$, Temmer M. ${ }^{2}$, Gopalswamy N. ${ }^{3}$ \\ ${ }^{1}$ George Mason University, USA \\ 2 University of Graz, Austria \\ ${ }^{3}$ Goddard Space Flight Center, USA
}

The overarching goal of the VarSITI/ISEST project is to understand the origin, propagation and evolution of solar transients through the space between the Sun an the Earth, and develop the prediction capability of space weather. During the fiveyear period of the ISEST project, significant process has been made in our understanding of Sun-Earth connection system and space weather prediction. STEREO observations have revealed the full kinematic evolution of a large number of CMEs continuously from the Sun to the Earth. Observations also reveal unprecedented details of the 3D morphological structure of CME ejecta as well as the shocks driven by the ejecta. CME-CME interaction and CME-CIR interaction are extensively studied. Advanced 3D MHD data-constrained simulations, covering either the entire Sun-to-Earth CME formation and evolution or CME heliospheric propagation, can now simulate realistic CME events and reproduce the observed structure and evolution to a good extent. Prediction of CME time of arrival at $1 \mathrm{AU}$ can now consistently reach an accuracy of within 10 hours on average between predicted and observed times. The ISEST project consists of six working groups and one long-term campaign activity. It holds workshops every year in different continents. It has a persistent WIKI website for ISEST events and catalogs. The project results in a special topical collection in the Journal of Solar Physics and a spin-off book. 


\section{List of Authors}

A

\author{
Abdu M.A. (37) \\ Adebesin B.O. $(1,15)$ \\ Adebiyi S.J. $(1,15)$ \\ Adeniyi J.O. (1, 15)
}

Akiyama S. $(13,14,23,45)$

Alexakis, P. (2)

Aminalragia-Giamini S. (17)

Angelov Chr. (29)

Angelov I. (29)

Areso O. (6)

Arsov T. (29)

Azeem I. (46)

B

Baker D. (16)
Balan N. (28)
Barros D. (11, 37)
Belakhovsky V.B. $(2,3)$
Belehaki A. $(3,38)$
Bhowmik Pr. (27)
Bogomolov A. (25)
Borisenko A.V. (4)
Borodkova N.L. (45)
Brun A.S. (4, 15)
Buchlin E. (15)
Buriti R.A. (11)
C
CDPP team (13)
Chavan G.A. (27)
Cherepanova L. (20)
Clette F. (5)

Daglis I.A. $(17,28)$

Danov D. (25)

Dasso S. $(5,6)$

Dayeh M.A. (7)

Dechev M. $(6,10,19,20,26)$

Dement'eva A. (20)
Demetrescu Cr. $(7,9)$

Desai M.I. (7)

Despirak I.V. $(8,14)$

Dickson E. (26)

Dikpati M. (9)

Dissauer K. $(20,26)$

Dobrica V. $(7,9)$

Dorotovič I. (30)

Duchlev P. $(6,10,19)$

Duggirala, P. (10)

Dumbovic M. $(11,37)$

E

Ebert R.W. (7)

Erokhin N.S. (35)

F

Fainshtein V.G. (46)

Falayi E. (32)

Feng X. (34)

Figueiredo C.A.O.B. $(11,37)$

Fonseca J.M. (30)

Fujuwara, H. (26)

Funke B. $(12,42)$

G

Gavrilov N.M. $(12,31)$

Génot V. (13)

Georgieva K. (7)

Gopalswamy N. (13, 14, 23, 40, 45, 47)

Greculeasa R. (9)

Gromov S.V. $(8,46)$

Gromova L.I. $(8,46)$

Guineva V. $(14,43)$

Gulisano A.M. (6)

H

Hazra S. (15)

Hoffmann L. (46)

Holzknecht L. (37)

Hori T. (3)

Horne R.B. (15) 
Hotta H. (16)

I

Iijima H. (16)

Ikubanni S.O. $(1,15)$

Imada S. (16)

IPIR team (25)

J

Jeni V.N. (27)

Jin H. (26)

$\mathbf{K}$

Kan L. $(43,44)$

Kanekal S. (16)

Karan D.K. (30)

Kasahara S. (3)

Kasahara Y. (2)

Kashapova L. $(25,26)$

Katsavrias Ch. $(17,28)$

Katsova M.M. (18)

Kaushik S. (18)

Kaushik S.C. (18)

Kazama Y. (3)

Keika K. (3)

Keran D.K. (10)

Khramova E. (30)

Kilcik A. (19, 34)

Kirov B. (7)

Kleimenova N. $(8,14)$

Koleva K. $(6,10,19,26)$

Kononova N. (21)

Koutroumbas K. (38)

Kozarev K. $(20,26)$

Kozelov B.V. (8)

Kozelova T.V. (8)

Krivolutsky A. (20, 21, 42)

Krivova N. (21)

Kshevetskii S.P. (12)

Kukhianidze V. (40)

Kukoleva A. (21)

Kusano K. $(16,22)$
$\mathbf{L}$

LAGO collaboration (6)

Laskar F.L. (10)

Lastovicka J. $(22,42)$

Lefèvre L. (5)

Lepping R.P. $(43,44)$

Li W. (17)

Liu H. (36)

Lodkina I.G. (45)

Loznikov V.M. (35)

Lubchich A.A. (8)

Lübken F.-J. $(22,42)$

Lühr H. (36)

Lynn H. (44)

M

Mäkelä P. (13, 14, 23, 45)

Malandraki O.E. (23)

Malysheva L.M. (8)

Mandal S. (10)

Manuilova R. (24)

Martens P.C. (24)

Matsuoka A. $(2,3)$

Mavromichalaki H. (2)

Medvedeva I.V. (31)

Michalek G. (23)

Mikhailovskaya L.A. (35)

Miller S.D. (46)

Miloch W.J. (25)

Miteva R. (19, 20, 25, 26, 33, 39)

Miyoshi Y. $(2,3,26)$

Monstein C. (40)

Mora A. (30)

Mungufeni P. (40)

Myagkova I. (25)

Myshyakov I. (25, 39)

$\mathbf{N}$

Nade D.P. (27)

Nakamura T. (42)

Nandi D. (27)

Narayanan V.L. (28) 


\author{
Nasi A. (28) \\ Nikolova N. (29) \\ Nikte S.S. (27) \\ Nosé, M. (29) \\ $\mathbf{0}$
}

Obridko V. $(30,41)$

Ogurtsov M. (41)

Okoh D.I. (32)

Otsuka Y. (11, 32, 35, 36, 37)

Özgüc A. (19)

$\mathbf{P}$

Papadimitriou C. (17)

Park J. (36)

Paulino I. $(11,37)$

Pawar R.P. (27)

Pereira M. (6)

Perminov V.I. (31)

Perri B. (15)

Pertsev N.N. (31)

Petrov N. $(25,39)$

Pires R. (30)

Podgorny A.I. $(4,31)$

Podgorny I.M. $(4,31)$

Podladchikova O. (17)

Popov A.A. (31)

Potdar S.S. (27)

PWING Team (35)

Q

Qian L. (32)

$\mathbf{R}$

Rabiu A.B. (32)

Ragulskaya M. (30)

Reville V. (15)

Riazantseva M.O. (45)

Ribeiro R. (30)

Roussev I.I. (33)

Rybák J. (30)

$\mathbf{S}$

Samwel S.W. $(26,33)$
Sandberg I. (17)

Sarp V. (34)

Segun B. (32)

Seppala A. (42)

Shen F. (34)

Shinagawa H. (26)

Shinohara I. $(2,3)$

Shiokawa K. (2, 3, 11, 32, 35, 36, 37)

Shiota D. (16)

Shkevov R. (35)

Sibeck D. (16)

Siingh D. (27)

Singh R.P. (10)

Stefan Cr. (9)

Stolle Cl. (42)

Straka III W. (46)

Strugarek A. $(4,15)$

Suzuki S. (36)

$\mathbf{T}$

Takahashi H. (11, 37)

Tam S. (3)

Taori A. (27)

TechTIDE EC consortium (3)

Temmer M. $(20,26,37,39,47)$

Tomikawa Y. (38)

Török, T. (38)

Tsagouri I. (38)

Tsironis C. (17)

Tsvetkov Ts. $(25,39)$

Tuniyants T. (20)

$\mathbf{U}$

Umuhire A.C. (40)

Uwamahoro J. (40)

V

Vadas Sh.L. (46)

Vashalomidze Z. (40)

Veretenenko S. (41)

Veronig A. $(20,26)$

Vorobeva Ek. (24)

Vrsnak B. (37) 


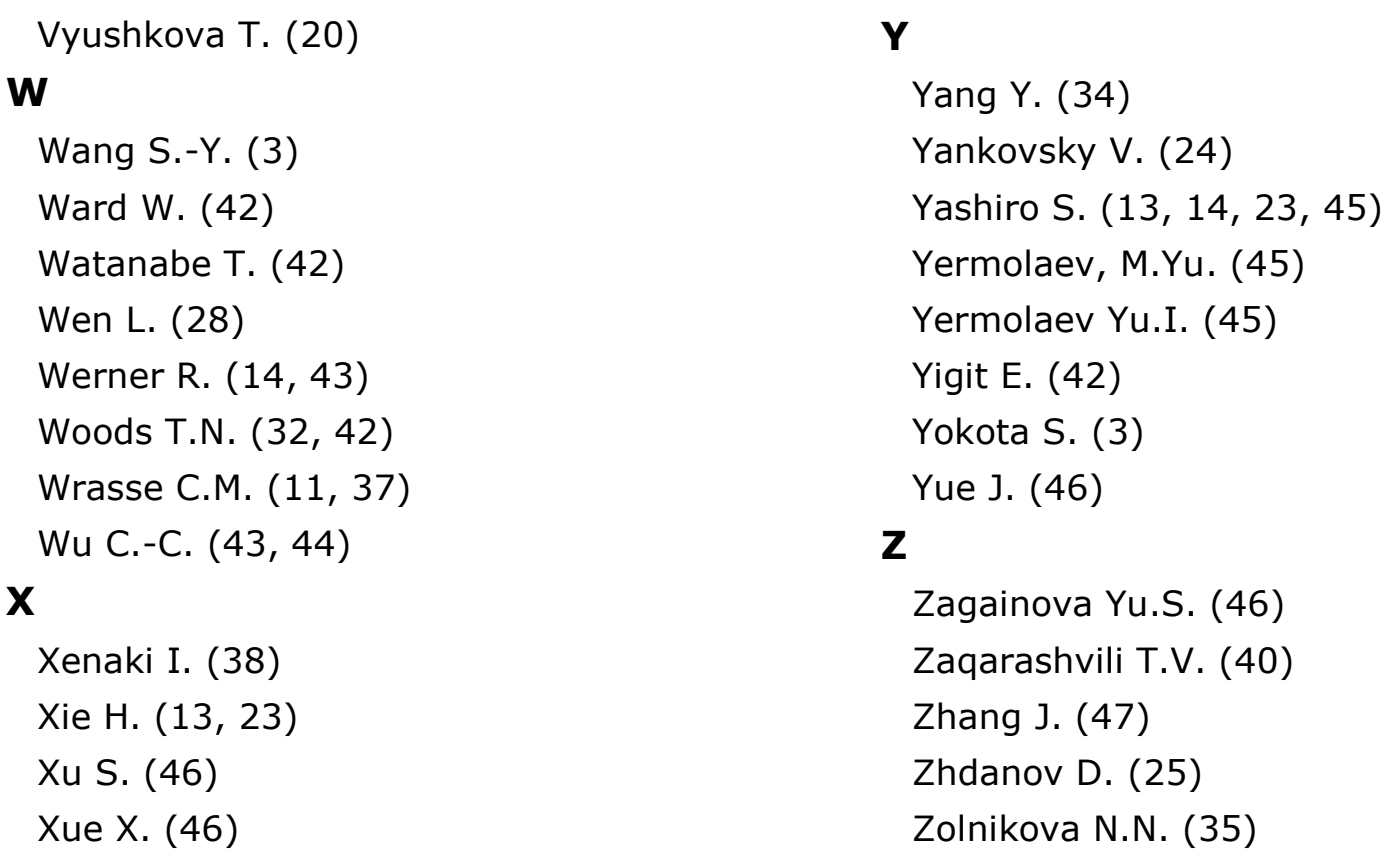

